\title{
Histone deacetylase 3 promotes innate antiviral immunity through deacetylation of TBK1
}

\author{
Jie-lin Tang ${ }^{1,2}$, Qi Yang ${ }^{1}$, Chong-hui Xu ${ }^{1,2}$, He Zhao ${ }^{1}$, Ya-ling Liu ${ }^{1,2}$, Can-yu Liu ${ }^{1,2}$, Yuan Zhou ${ }^{1}$, Dong-wei Gai ${ }^{1,2}$, \\ Rong-juan Pei ${ }^{1}$, Yun Wang ${ }^{1}$, Xue Hu ${ }^{1}$, Bo Zhong ${ }^{3}$, Yan-yi Wang ${ }^{1}$, Xin-wen Chen ${ }^{1,4 \bowtie}$, Ji-zheng Chen ${ }^{1 \bowtie}$ \\ ${ }^{1}$ State Key Laboratory of Virology, Wuhan Institute of Virology, Center for Biosafety Mega-Science, Chinese Academy of \\ Sciences, Wuhan 430071, China \\ 2 University of Chinese Academy of Sciences, Beijing 100049, China \\ ${ }^{3}$ Medical Research Institute, School of Medicine, Wuhan University, Wuhan 430071, China \\ ${ }^{4}$ Guangzhou Institutes of Biomedicine and Health, Chinese Academy of Sciences, Guangzhou 510530, China \\ $\square$ Correspondence: chenxw@wh.iov.cn (X. Chen), chenjz@wh.iov.cn (J. Chen) \\ Received April 12, 2020 Accepted June 17, 2020
}

\begin{abstract}
TANK-binding kinase 1 (TBK1), a core kinase of antiviral pathways, activates the production of interferons (IFNs). It has been reported that deacetylation activates TBK1; however, the precise mechanism still remains to be uncovered. We show here that during the early stage of viral infection, the acetylation of TBK1 was increased, and the acetylation of TBK1 at Lys241 enhanced the recruitment of IRF3 to TBK1. HDAC3 directly deacetylated TBK1 at Lys241 and Lys692, which resulted in the activation of TBK1. Deacetylation at Lys241 and Lys692 was critical for the kinase activity and dimerization of TBK1 respectively. Using knockout cell lines and transgenic mice, we confirmed that a HDAC3 null mutant exhibited enhanced susceptibility to viral challenge via impaired production of type I IFNs. Furthermore, activated TBK1 phosphorylated HDAC3, which promoted the deacetylation activity of HDAC3 and formed a feedback loop. In this study, we illustrated the roles the acetylated and deacetylated forms of TBK1 play in antiviral innate responses and clarified the post-translational modulations involved in the interaction between TBK1 and HDAC3.
\end{abstract}

Electronic supplementary material The online version of this article (https://doi.org/10.1007/s13238-020-00751-5) contains supplementary material, which is available to authorized users.
KEYWORDS TBK1, HDAC3, deacetylation, IRF3, innate immune

\section{INTRODUCTION}

The detection of pathogen-associated molecular patterns (PAMPs) on microbial pathogens via pattern-recognition receptors (PRRs) is the first step in eliciting innate immune responses in the host. The activation of PRRs triggers downstream signaling cascades that lead to the rapid production of type I interferons (IFNs) and proinflammatory cytokines that eliminate pathogens (Akira et al., 2006; Medzhitov, 2007; Takeuchi and Akira, 2010). Post-translational modifications (PTMs) act as vital regulators of cellular signal transduction systems during innate immune responses. Lysine acetylation, which affects the stability, activation, and subcellular localization of proteins, as well as the interaction of proteins with other proteins or DNA, has been identified as a critical regulator of the innate immune system (Mowen and David, 2014; Li et al., 2016; Dai et al., 2019). Several non-histone signal transducing proteins, which are important for TLR pathways, and transcriptional regulators involved in the expression of inflammatory cytokines are regulated by acetylation (Cao et al., 2008). However, the crosstalk that occurs between acetylation, conventional PTMs, and modulation of the kinase activity of signaling transducers in the innate immune response is still unknown.

Interferon regulatory factor 3 (IRF3) acts as a master transcription factor responsible for the production of IFN- $\beta$ $($ IFN- $\beta)$ and is essential for the establishment of innate 
immunity (Honda and Taniguchi, 2006). TBK1 is a key kinase for IRF3 activation that leads to the expression of type I IFNs (Fitzgerald et al., 2003). Several positive or negative regulatory mechanisms for the control of TBK1mediated antiviral responses have been described (An et al., 2015; Song et al., 2016; Liu et al., 2017). The activation and function of TBK1 are orchestrated by ubiquitination, dimerization, and phosphorylation (Li et al., 2011; Tu et al., 2013). Seven lysine residues on TBK1 have been shown to be modified by acetylation, and Lys 241 deacetylation is critical for the kinase activity of TBK1 (Li et al., 2016). However, the precise mechanism still remains to be uncovered.

Epigenetic regulation is important for innate immune responses, especially for the modulation of signaling regulators, the transcription of genes encoding inflammatory products, and PTMs required for the activation of signaling pathways (Smale et al., 2014; Li et al., 2016). HDAC3, which belongs to the Class I HDACs, is thought to be a classical histone deacetylase, and non-histone targets have been reported (Mihaylova et al., 2011). Although the regulation of HDAC1, 4, 6, 8 or 9 in innate immunity has been revealed ( $\mathrm{Li}$ et al., 2016; Meng et al., 2016; Yang et al., 2018), little is unknown about the role HDAC3 plays in anti-microbial innate immunity. Moreover, the phosphorylation of HDAC3 promotes its deacetylase activity, but the kinase for HDAC3 is unknown (Zhang et al., 2005).

Here, we report that HDAC3 acts as a positive regulator in antiviral immune responses. HDAC3 deficiency suppressed the production of IFN- $\beta$, a type I IFN, both in vitro and in vivo. HDAC3 deacetylated TBK1 to promote the dimerization and activation of TBK1. The acetylation of TBK1 at Lys241 during the early stage of viral infection enhanced the recruitment of IRF3 to TBK1. Furthermore, activated TBK1 further phosphorylated HDAC3 to enhance the activity of deacetylated HDAC3 and activate TBK1. Our results provide insights into the PTMs involved in the interaction between TBK1 and HDAC3 during antiviral innate responses.

\section{RESULTS}

\section{HDAC3 positively regulates type I IFN production}

In a shRNA library screen, we found that knockdown of HDAC3 impaired Sendai virus (SeV)-induced activation via the IFN- $\beta$ (IFNB1) gene promoter. To verify this phenomenon, two shRNA-mediated knockdown of HDAC3 was performed in HEK293T cells. HDAC3 knockdown reduced the promoter activity of IFN- $\beta$ and IFN-stimulated response elements (ISRE) (Fig. 1A). In addition, after stimulation with various inducers, including $\mathrm{SeV}$, vesicular stomatitis virus (VSV), and herpes simplex virus (HSV-1), IFNB1 production in the HDAC3-knockdown samples was significantly lower compared to the control samples (Fig. 1B). Similarly, knockdown of HDAC3 using siRNA validated these results (Fig. S1A and S1B). We further generated HDAC3-deficient THP-1 cells by CRISPR/Cas9 in order to investigate whether
Figure 1. HDAC3 positively regulates type I interferon production. (A) Luciferase activity in shNC, shHDAC3-1 or shHDAC3-2 cell lines $\left(1 \times 10^{5}\right)$, transfected for $36 \mathrm{~h}$ with a luciferase reporter for IFN- $\beta$ (IFN- $\beta$-luc) or for ISRE (ISRE-luc), uninfected or infected with SeV for $10 \mathrm{~h}$ before luciferase assays were performed. HDAC3-Knockdown efficiency in the shHDAC3 cell lines were confirmed by immunoblotting analysis (lower). (B) ELISA analysis of IFN- $\beta$ in the supernatant of shNC, shHDAC3-1 cell lines, followed by infection with or without SeV, VSV, HSV-1. (C) ELISA of IFN- $\beta$ in the supernatant of THP-1 HDAC3deficient cells (HDAC3 KO-1, HDAC3 KO-2), followed by infection with different stimulus (as shown). HDAC3 knockout efficiency in the THP-1 cells were confirmed by immunoblotting analysis (right). (D) Luciferase activity assay in HEK293T cells $\left(1 \times 10^{5}\right)$, co-transfected for $36 \mathrm{~h}$ with empty vectors, increased amounts of HDAC 3 expression plasmids or Flag tagged HDAC3-H134Q (100 ng) together with a luciferase reporter for IFN- $\beta$ (IFN- $\beta$-luc) or for ISRE (ISRE-luc), followed by infection with or without $\mathrm{SeV}$ for $10 \mathrm{~h}$ before luciferase assays were performed. (E) ELISA analysis of IFN- $\beta$ in the supernatant of HEK293T cells $\left(1 \times 10^{5}\right)$, transfected for $36 \mathrm{~h}$ with empty vectors, increased amounts of HDAC 3 expression plasmids or Flag tagged HDAC3-H134Q (100 ng), followed by infection with or without SeV for $10 \mathrm{~h}$. (F) ELISA of IFN- $\beta$ analysis in the supernatant of HDAC3-stable-expressing THP-1 cells, followed by infection with different stimulus (as shown). Data are representative of three independent experiments. Graphs show mean $\pm \mathrm{SD} ; n=3$. * $P<0.05$; * $P<0.01 ;{ }^{* \star} P<0.001$ (Student's $t$-test).

HDAC3 was required for virus-induced type I IFN responses in immunocytes. The secretion of IFN- $\beta$ from HDAC3-deficient THP-1 cells was impaired as compared with their secretion from the control cells after stimulation by various inducers, including RNA viruses (SeV and VSV), DNA virus (HSV), synthetic RNA duplex poly (I:C), bacterial lipopolysaccharide (LPS), cGAMP, or CpG oligodeoxynucleotide (ODN) (Fig. 1C). We obtained similar results in vitro using a macrophage cell line (RAW264.7) with HDAC3 knockdown (Fig. S1C). These results indicated specific involvement of HDAC3 in the expression of type I IFNs.

In order to further confirm the contribution of HDAC3 to antiviral responses, we stimulated HDAC3-overexpressing HEK293T cells with SeV. The promoter activity of IFNB1 or ISRE and the production of IFN- $\beta$ were significantly higher when HDAC3 was overexpressed as compared to samples transfected with the empty vector rather than HDAC3 deacetylase inactive mutant $(\mathrm{H} 134 \mathrm{Q})$, and the agonists occurred in a dose-dependent manner after infection with $\mathrm{SeV}$ (Fig. 1D and 1E). Accordingly, the production of IFN- $\beta$ was higher in HDAC3-overexpressing THP-1 cells stimulated with various inducers (Fig. 1F). Together these results 


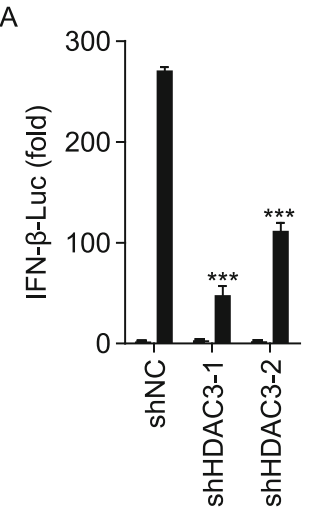

HDAC3

GAPDH

C

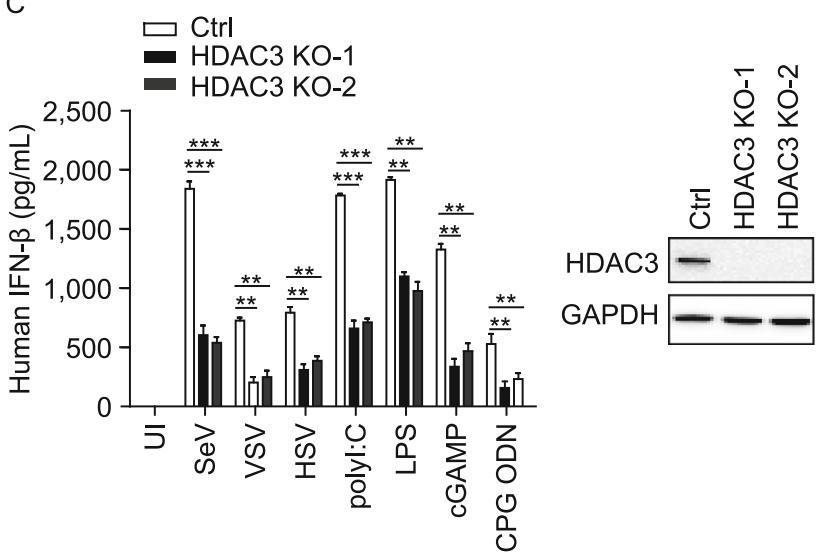

D
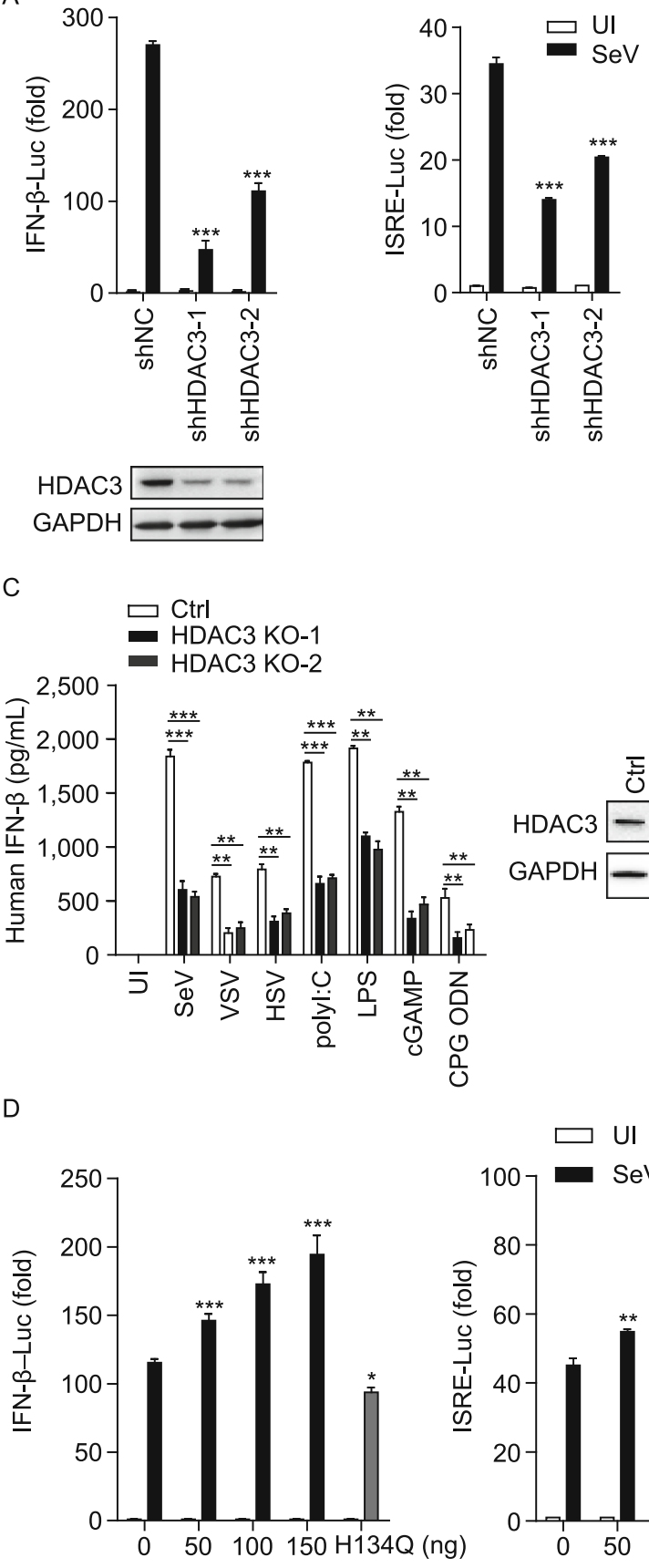

B $\square \operatorname{shNC}$

- shHDAC3-1

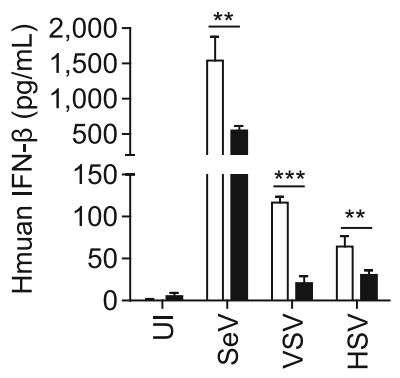

$\mathrm{F}$

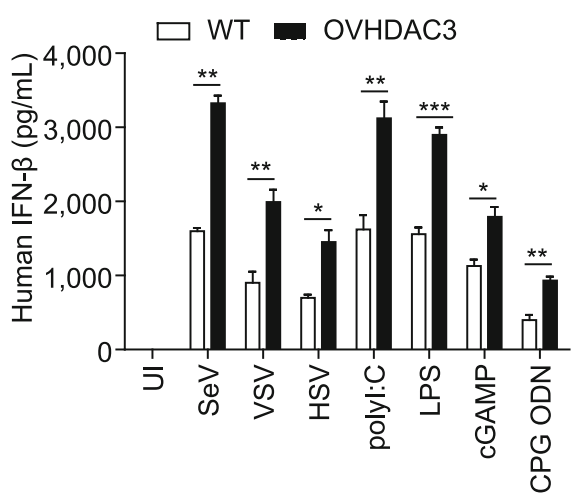

$\mathrm{E}$
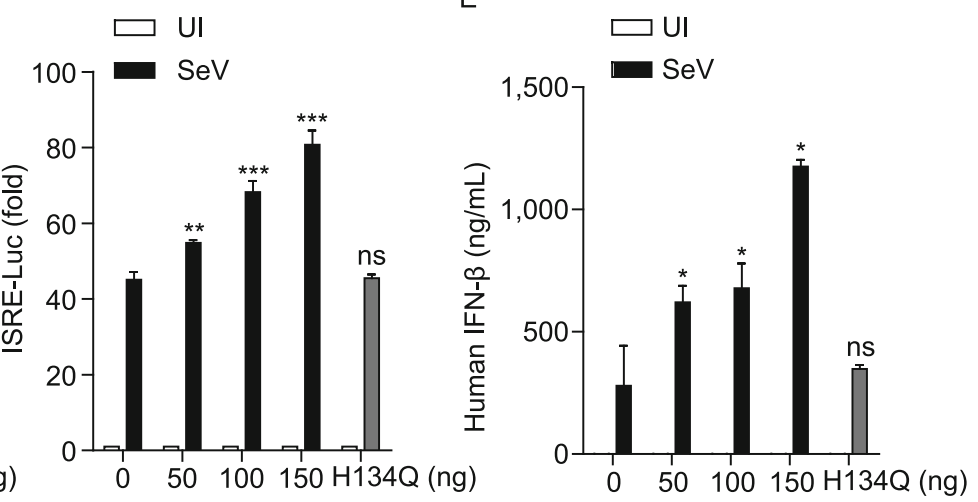


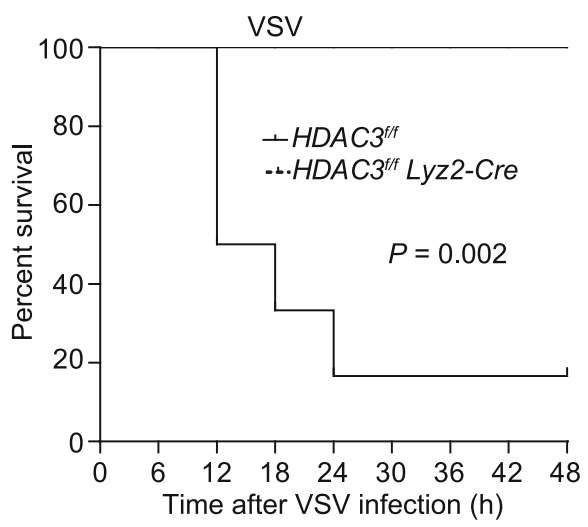

B

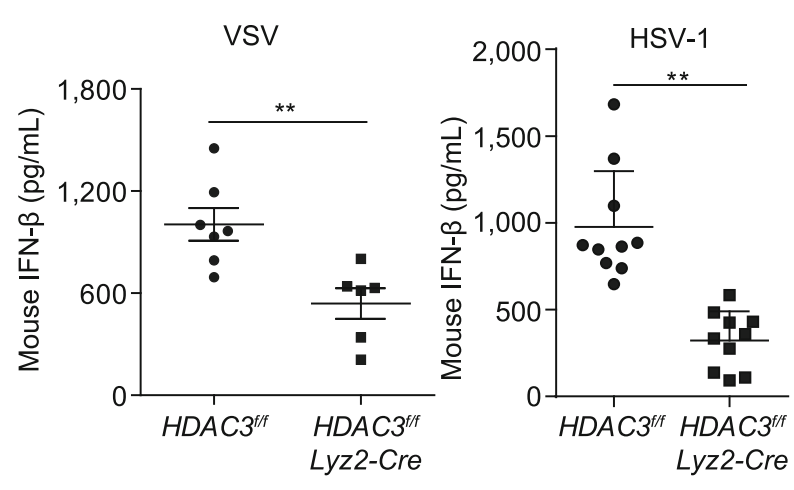

D
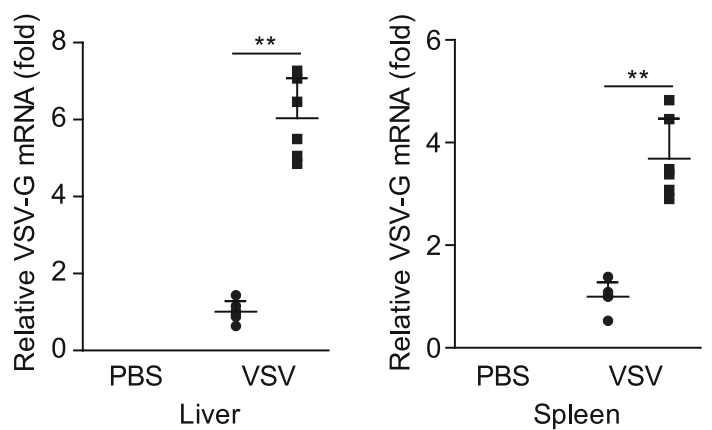

C
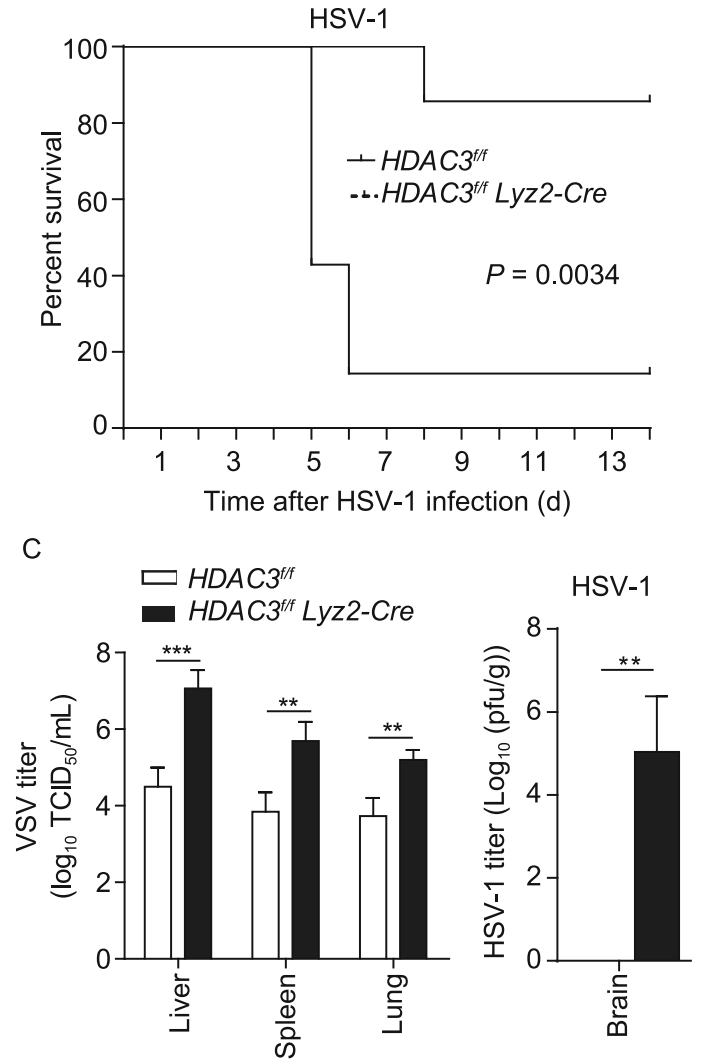

- $H_{D A C 3^{f f f}}$

- HDAC3 $3^{\text {sf }} L y z 2-C r e$
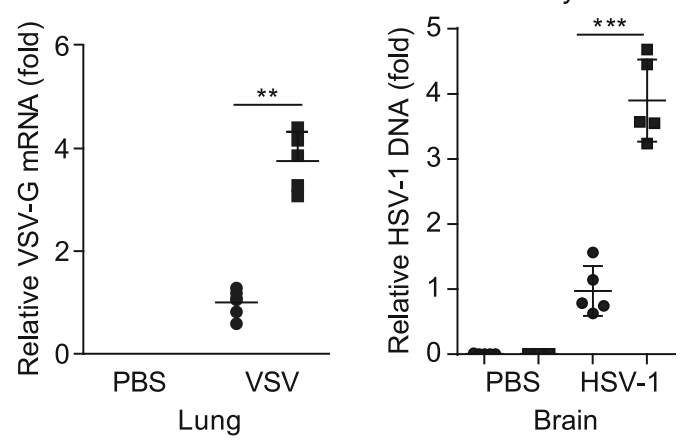

E

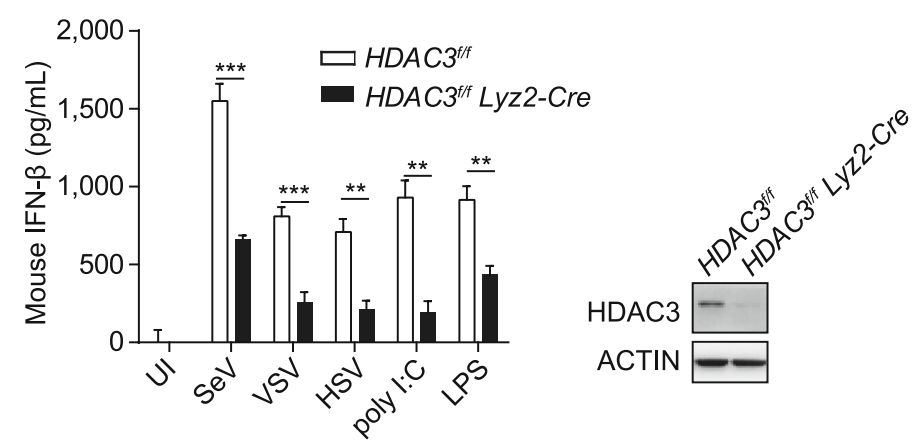


Figure 2. Pivotal role for HDAC3 in the antiviral response in vivo. (A) Survival (Kaplan-Meier curve) of 8-week-olds $\operatorname{HDAC3}^{f l / f l}(n=5,7)$ and HDAC $3^{f l f l}$ Lyz2-Cre mice $(n=5,7)$ intraperitoneal injected with VSV-GFP or HSV-1-GFP $\left(7 \times 10^{7}\right.$ $\mathrm{PFU} / \mathrm{g}, 2 \times 10^{7} \mathrm{PFU} / \mathrm{g}$ ) [plaque-forming unit] monitored for $48 \mathrm{~h}$ or $2 \mathrm{w}$. (B) ELISA analysis of IFN- $\beta$ in the sera from $H D A C 3^{f l / f l}$ $(n=7,10)$ and $H D A C 3^{f l f f l}$ Lyz2-Cre mice $(n=6,10)$ after VSV or HSV-1 infection. (C) VSV load in the liver, spleen, lungs or HSV load in the brain of $H D A C 3^{f l / f l}$ or $H D A C 3^{f l / f l} L y z 2-C r e$ mice $(n=5$ per group) after intraperitoneal infection with VSV (24 h) (as in left) or HSV-1 (5 days) (as in A), assessed by endpoint-dilution assay and presented as $50 \%$ tissue culture infectious dose $\left(T_{C I D}\right)$. (D) Quantitative PCR analysis of VSV RNA in the liver, spleen, lungs or HSV-1 DNA in the brain of $H D A C 3^{f l f f l}$ or $H D A C 3^{\text {fl/fl }} L y z 2-C r e$ mice after intraperitoneal injection of PBS or VSV (24 h) or HSV-1 (5 d) (as in A). (E) ELISA analysis of IFN- $\beta$ (left) in the supernatant of HDAC $3^{f l f l}$ or $H D A C 3^{f / f l} L y z 2-$ Cre bone marrow derived macrophages (BMDMs), infected with SeV, VSV, HSV-1, poly (I:C) and LPS. HDAC3-deficiency in BMDMs were confirmed by immunoblotting analysis (right). Data are representative of three independent experiments. Graphs show mean $\pm \mathrm{SD}$; ${ }^{* *} P<0.01 ;{ }^{* *} P<0.001$ (Student's $t$ test).

suggested a positive role for HDAC3 in PRR-induced induction of type I IFN in various cell types.

\section{Pivotal role for HDAC3 in the antiviral response in vivo}

To further elucidate the importance of HDAC3 in antiviral immunity in vivo, we adopted a strategy to generate $H D A C 3$ flox/flox-Lyz2-Cre mice (HDAC3 ${ }^{\text {fl/fl }}$ Lyz2-Cre), which undergo deletion of loxP-flanked HDAC3 alleles $\left(H D A C 3^{f l f f}\right.$ ) specifically in myeloid cells via Cre recombinase expressed from the myeloid cell-specific gene $L y z 2$ ( $L y z 2-C r e)$. We then challenged these mice with VSV or HSV and found that the mortality rate of $H D A C 3^{f l f l} L y z 2-C r e$ was greater than that of the $H D A C 3^{f l f l}$ littermates (Fig. 2A). HDAC3-deficient mice produced decreased levels of serum IFN- $\beta$ in response to infection with VSV or HSV than their $H D A C 3^{\text {fl/fl }}$ littermates (Fig. 2B). The VSV titers and replication in various organs and HSV titers and replication in the brain were also significantly greater in HDAC3-deficient mice than in their $H D A C 3^{\text {flffl }}$ counterparts (Fig. $2 \mathrm{C}$ and 2D). The secretion of IFN- $\beta$ from HDAC3-deficient bone marrow-derived macrophages (BMDMs) from HDAC ${ }^{f l f l}$ Lyz2-Cre mice was severely impaired as compared with the secretion from $H D A C 3^{\text {fl/fl }}$ BMDMs stimulated by various inducers, including SeV, VSV, HSV-1, poly (I:C), or (LPS) (Fig. 2E). Therefore, HDAC3 was required for the efficient production of type I IFNs and for the resistance of these mice to viral infection.
Deficiency in HDAC3 impairs virus-induced phosphorylation of IRF3

In order to understand the mechanism by which HDAC3 regulates innate antiviral signaling, we screened the signaling pathways responsible for initiating the production of IFN$\beta$. We investigated if HDAC3 influenced the levels of PRRtriggered signaling molecules using luciferase reporter assays. Knockdown of HDAC3 significantly inhibited IFNB1 reporter activity induced by upstream activators, including RIG-I, VISA, and TBK1, but not IRF3 or IRF3-5D (Fig. 3A). Phosphorylation of the transcription factor IRF3 at Ser386 has been shown to result in oligomerization, which has been strongly correlated with its full activation (Suhara et al., 2000). We found reduced phosphorylation of IRF3 at Ser386 and TBK1 at Ser172 induced by SeV in HDAC3-knockdown HEK293T cells, HDAC3-deficient THP-1 cells, or HDAC3deficient BMDMs (HDAC3 $\left.{ }^{f l f l} L y z 2-C r e\right)$ than in their control counterparts (Fig. 3B-D). Similarly, dimerization of IRF3 was weakened greatly in HDAC3-deficient THP-1 cells infected with $\mathrm{SeV}$ as compared to control THP-1 cells (Fig. 3E). Simultaneously, the results of immunoprecipitation showed that the interaction of TBK1 with VISA, TRAF6, or TANK had minimal enhancement when HDAC3 was present (Fig. S2AC). These data indicated that HDAC3 might be involved in the IRF3-mediated innate immune response by targeting TBK1 and affecting the activation of IRF3.

In order to investigate whether TBK1 was able to interact with HDAC3, we overexpressed HA-tagged TBK1 and Flag-tagged HDAC3 together in HEK293T cells. Immunoprecipitation and immunoblot assays indicated that HDAC3 and TBK1 associated together (Fig. 3F). The immunofluorescence detection showed co-localization of HDAC3 and TBK1 in HEK293T cells (Fig. S3A). Endogenous IP analysis suggested that HDAC3 constitutively interacted with TBK1 during SeV infection (Fig. 3G). It has been reported and our experiments revealed that HDAC3 shuttles between the nucleus and cytoplasm (Fig. S3B) (Chini et al., 2010). We constructed HDAC3 mutants that predominantly localize to cytoplasm (HDAC3- $\triangle$ NLS) and nucleus (2×NLSHDAC3), respectively (Fig. S3C) (Park et al., 2014). As expected, the phosphorylation of TBK1 and IRF3 was enhanced with the expression of wild-type HDAC3 or cytoplasmic HDAC3 (HDAC3- $\triangle$ NLS), but not the nuclear HDAC3 (Fig. S3D). The HDAC3- $\triangle$ NLS, not HDAC3-NLS, could promote the IFN-beta mRNA level and the production of IFN-beta during $\mathrm{SeV}$ challenge, indicating that the cytoplasmic HDAC3 that promotes activation of IRF3 (Fig. S3E and S3F). These results implied that regulation HDAC3 gene transcription had little effect on activation of IRF3. Collectively, these data suggested that HDAC3 could interact with TBK1 to promote the phosphorylation and activation of IRF3 by TBK1. 

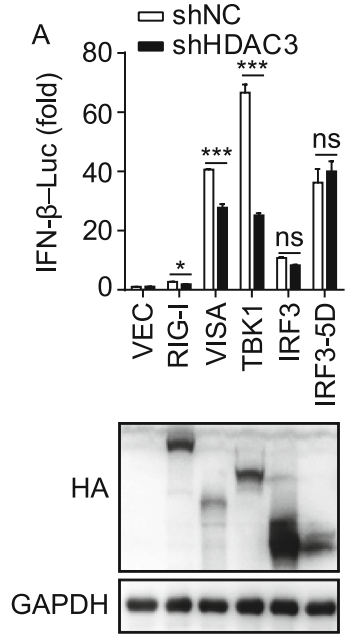

F
B

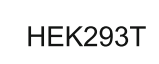
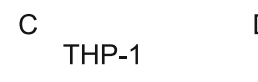

BMDMs

E
p-IRF3 (386)

IRF3

HDAC3

GAPDH

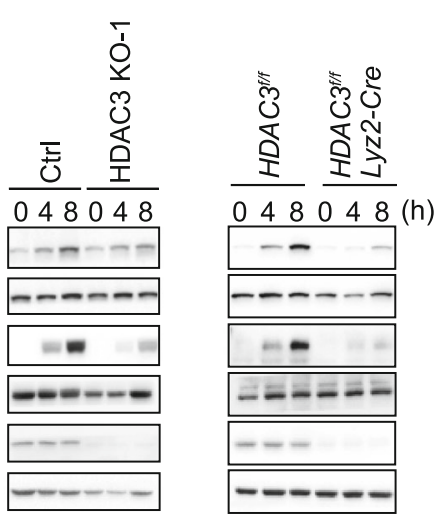

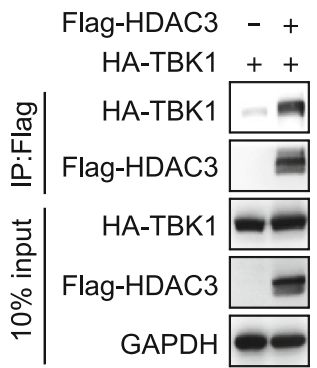

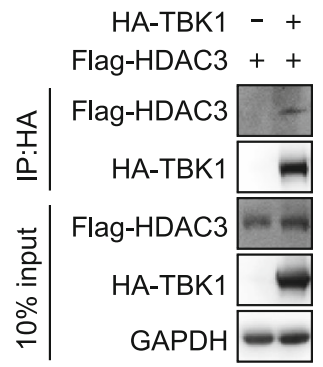

G

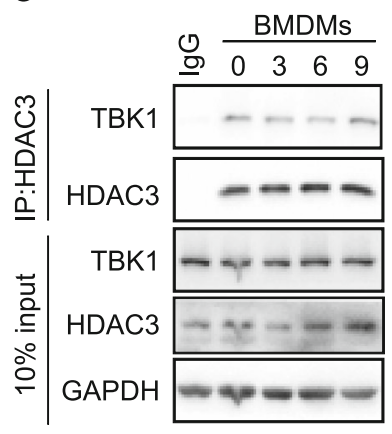

Figure 3. Deficiency of HDAC3 impairs phosphorylation of IRF3 by TBK1. (A) Luciferase activity (upper) in shNC or shHDAC3 cell lines $\left(1 \times 10^{5}\right)$, transfected with an IFN- $\beta$-Luc, together with control vector (VEC), HA-tagged RIG-I, VISA, TBK1, IRF3, or IRF3$5 \mathrm{D}$; the protein expression of RIG-I, VISA, TBK1, IRF3, or IRF3-5D were confirmed by immunoblotting analysis (lower). (B-D) Immunoblot analysis of phosphorylated and total TBK1 or IRF3 in shHDAC3 HEK293T cell lines (B), THP-1 HDAC3 deficient cell lines (C), $H D A C 3^{f l / f l}$ or $H D A C 3^{\text {fl/fl } L y z 2-C r e ~ B M D M s ~(D), ~ f o l l o w e d ~ b y ~ i n f e c t i o n ~ w i t h ~ S e V ~ f o r ~ t h e ~ i n d i c a t e d ~ t i m e s . ~(E) ~ I m m u n o b l o t ~ a n a l y s i s ~ o f ~}$ IRF3 in dimerization in HDAC3-deficient THP-1 cells and left infected with SeV for the indicated times, followed by native PAGE. $(F)$ The interaction between HDAC3 and TBK1. HEK293T cells $\left(1.5 \times 10^{6}\right)$ were co-transfected with the indicated plasmids $(3 \mu \mathrm{g}$ each) for $48 \mathrm{~h}$. Co-immunoprecipitation and immunoblotting were performed with the indicated antibodies. (G) Endogenous HDAC3 dynamically interacts with TBK1, followed by infection with SeV. BMDMs were left uninfected or infected with SeV for the indicated times. Co-immunoprecipitation and immunoblotting were performed with the indicated antibodies. Data are representative of three independent experiments. Graphs show mean $\pm \mathrm{SD} ; n=3$. ns, no significant differences; $P<0.05 ;{ }^{* \star *} P<0.001$ (Student's $t$-test).

HDAC3 directly enhances the kinase activity of TBK1 via its deacetylase activity

To analyze the mechanistic details, we first considered whether the deacetylation activity of HDAC3 played a critical role in inhibiting TBK1-mediated IRF3 activation. A reporter assay indicated that RGFP966, a specific inhibitor of HDAC3, could impair IFNB1 transcriptional activity and the phosphorylation of IRF3 at Ser386 and TBK1 at Ser172 in a dose-dependent manner during SeV infection (Fig. 4A and B). Furthermore, we mapped the domain of HDAC3 that interacted with TBK1. An HDAC3 mutant consisting of the HDAC domain alone was still capable of interacting with TBK1 (Fig. 4C). To determine how TBK1 interacted with HDAC3, we constructed TBK1 mutants with deletions in various domains and found that the kinase domain and coiled-coil domain of TBK1 were required for the interaction of TBK1 with HDAC3 (Fig. 4D).

To further investigate the effect of HDAC3 on the regulation of the kinase activity of TBK1, we performed an in vitro TBK1 kinase assay using a TBK1 kinase enzyme system, with or without HDAC3. As expected, HDAC3 markedly increased TBK1 kinase activity (Fig. 4E). We also introduced the fusion proteins containing HDAC3 (Flag-HDAC3) or recombinant HDAC3, Flag-tagged TBK1 and HA-tagged IRF3, or purified TBK1 and IRF3 protein into the in vitro kinase assay. Immunoblot (IB) analysis of IRF3 phosphorylation at Ser386 and TBK1 phosphorylation at Ser172 revealed that the phosphorylation of IRF3 or TBK1 was 
enhanced in the presence of HDAC3 (Fig. 4F). These results indicated that the deacetylase activity of HDAC3 could positively regulate TBK1 kinase activity.

\section{Lys241 and Lys692 deacetylation mediated by HDAC3 are critical for TBK1 kinase activity}

We assessed the lysine acetylation of endogenous TBK1 immunoprecipitated from HEK293T cells in the absence of stimuli and found that endogenous TBK1 was distinctly acetylated (Fig. 5A). The acetylation of TBK1 was reduced dramatically following HDAC3 overexpression (Fig. 5B). Correspondingly, TBK1 acetylation gradually increased when the cells were treated with the HDAC3 specific inhibitor RGFP966 (Fig. 5C). To identify definitively acetylated lysine residues in TBK1, we used an antibody against TBK1 to purify endogenous TBK1 from whole cell extracts of HDAC3deficient HEK293T cells infected with SeV. According to the reports and mass spectrometry analysis revealed that nine lysine residues of TBK1 were modified by acetylation: Lys30, Lys154, Lys236, Lys241, Lys251, Lys607, Lys646, Lys691, and Lys692 (Fig. 5D), all of which were distributed either in the kinase domain or the coiled-coil domain of TBK1 $(\mathrm{Li}$ et al., 2016). Both of these domains were the key domains through which TBK1 interacted with HDAC3. We then sought to determine whether the acetylation sites of TBK1 noted above were able to regulate the kinase activity of TBK1. Each of the identified lysine residues was mutated into a glutamine ( $K$ to $Q$ ) or arginine ( $K$ to $R$ ) (to mimic the acetylated or non-acetylated state of the lysine residue), followed by transient transfection and reporter assays. Compared to other mutations, the $\mathrm{K} 241 \mathrm{Q}$ and $\mathrm{K} 692 \mathrm{Q}$ mutants had an impaired ability to activate the IFN- $\beta$ reporter, whereas the K241R and K692R mutants still retained partial ability to activate the IFN- $\beta$ luciferase reporter (Fig. 5D). In parallel experiments, the $\mathrm{K} 241 \mathrm{Q}$ or $\mathrm{K} 692 \mathrm{Q}$ mutants showed reduced phosphorylation of IRF3, but the K241R or K692R mutants had a limited impact compared to the wild type (Fig. 5E). An in vitro TBK1 kinase assay using a TBK1 Kinase Enzyme System showed a higher luciferase value, indicating increased kinase activity in K241R and K692R mutants as compared to the $\mathrm{K} 241 \mathrm{Q}$ and $\mathrm{K} 692 \mathrm{Q}$ mutants (Fig. 5F). We then found that the $\mathrm{K} 241 \mathrm{Q}$ or $\mathrm{K} 692 \mathrm{Q}$ mutants had stronger interactions with HDAC3 than the K241R or K692R mutants (Fig. 5G). To further confirm the role of acetylation at Lys241 and Lys692, we generated specific antibodies against TBK1 acetylated at Lys241 or Lys692. Dot-blot analysis or Western blot indicated that the antibodies specifically recognized TBK1 peptide acetylated at Lys241 or Lys692, but did not recognize the control peptide or the K241R or K692R mutants (Fig. S4A-C). After transfecting the Flag-tagged TBK1, we found Lys241 or Lys692 acetylated TBK1, and the acetylation of TBK1 at Lys241 or Lys692 was increased in HDAC3-knockdown HEK293T cells and reduced in HDAC3 overexpressed HEK293T cells (Fig. 5H and 5I). Thus,
HDAC3 mediated the deacetylation of TBK1 at Lys241 and Lys692 to promote TBK1 kinase activity.

\section{Lys241 is critical for the interaction between TBK1 and IRF3}

By using a specific antibody against acetylated lysine residues, we analyzed Lys241-acetylated TBK1 immunoprecipitated from HEK293T cells during SeV infection. We detected that TBK1 was acetylated at Lys241 in HEK293T cells. We also found that the acetylation of TBK1 at Lys241 was strengthened and then weakened, and the level of acetylation was highest 3 hours post-infection with SeV (Fig. 6A). In an in vitro deacetylation assay, HDAC3 dramatically deacetylated TBK1 at Lys241 as compared to the control (Fig. 6B). In an in vitro kinase assay, we found that the K241R TBK1 mutant was better at mediating the in vitro phosphorylation of recombinant IRF3 (Fig. 6C). Thus, deacetylation of Lys241 was critical for the kinase activity of TBK1. Lys241 is located at the outer of the "pocket" of the kinase domain (Fig. 6D). We surmised that Lys241 could affect substrate binding, such as IRF3. To test this hypothesis, we analyzed the interaction between K241R or K241Q with IRF3. As shown in Fig. 6E, K241Q had a stronger interaction with IRF3 as compared to K241R. Together, these results suggested that the deacetylation of Lys 241 in TBK1 was important for its interaction with IRF3 and its kinase activity.

\section{Lys692 deacetylation controls the dimerization of TBK1}

We also analyzed acetylation of TBK1 at Lys692 during SeV infection. We found that the acetylation of TBK1 at Lys692 was strengthened and then weakened similar to Lys241, and the level of acetylation was highest at 3-5 hours post-infection with SeV (Fig. 7A). In an in vitro deacetylation assay, HDAC3 dramatically deacetylated TBK1 at Lys692 as compared to the control (Fig. 7B). Lys692 is located in the coiledcoil domain of TBK1, which is exclusively responsible for the association with the adaptor proteins. Not surprisingly, HDAC3 promoted the interaction between TBK1 and TRAF3 or $A Z I 2$, the adaptor proteins of TBK1 (Fig. 7C). Indeed, knockout of HDAC3 had the opposing effects (Fig. S5A and S5B). However, K692R or K692Q showed no different interaction with IRF3 as compared to the wild-type (Fig. 6E). We found that the dimerization of TBK1 was lower in $H_{D A C 3^{f l f l}} L y z 2-C r e$ peritoneal macrophage after infection with VSV (Fig. 7D). The dimerization was enhanced in $\mathrm{K} 692 \mathrm{R}$, but was reduced in K692Q as compared to the wildtype (Fig. 7E). To investigate the role of Lys692 deacetylation in dimerization, we constructed the mutants TBK1-355A/ 357A/547D to prevent dimerization of TBK1 (Larabi et al., 2013). When the dimerization was abolished, the acetylation of Lys692 was increased, but not the acetylation of Lys241 (Fig. S6A and S6B). Together, these results indicated that 
A

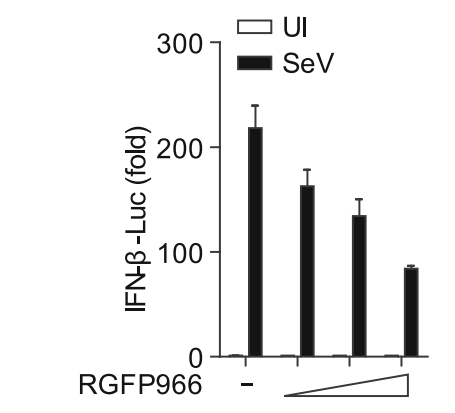

C

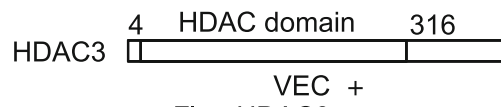

Flag-HDAC3 $(1-316)+$

Flag-HDAC3 (317-428)
B

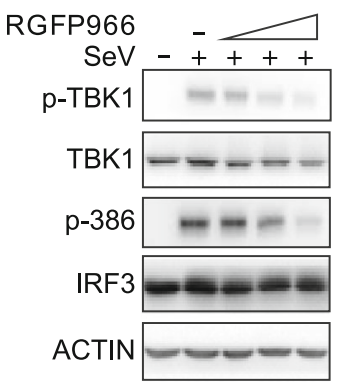

D

TBK1 (WT)

TBK1 (KD + ULD)

TBK1 (KD)

TBK1 (CC)

TBK1 (ULD + CC)

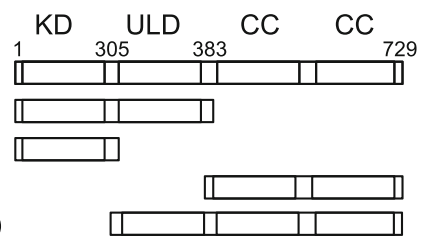

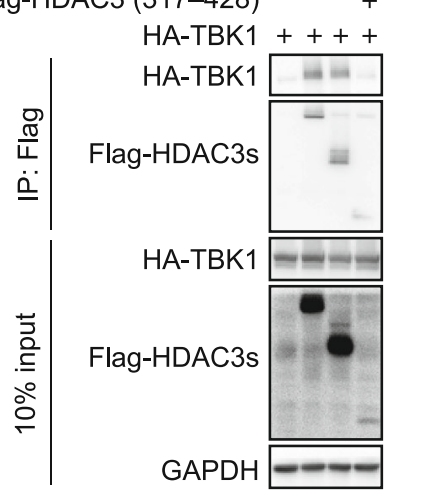

E

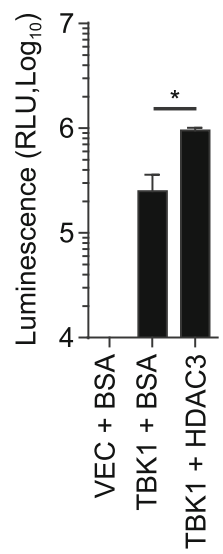

\section{EGFP-C1 +}

EGFP-C1-TBK1 (WT)

EGFP-C1-TBK1 (KD + ULD)

EGFP-C1-TBK1 (KD)

EGFP-C1-TBK1 (CC)

EGFP-C1-TBK1 (ULD + CC)

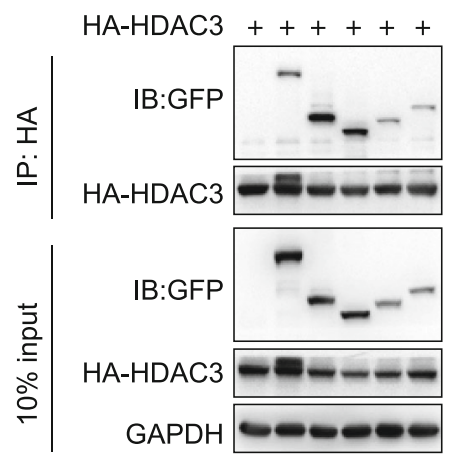

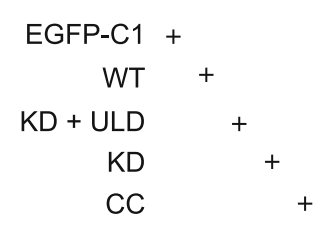

ULD + CC +

HA-HDAC3 ++++++

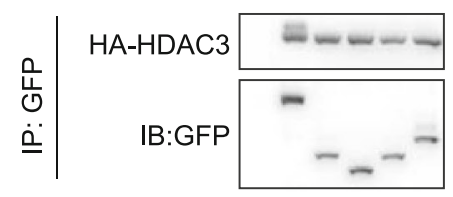

HA-HDAC3 $=\mathbf{E = - 1}$

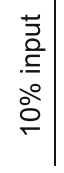

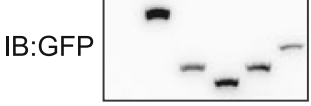

GAPDH ------

F
Flag-TBK1 -++
HA-IRF3 + + +
Flag-HDAC3 - - +

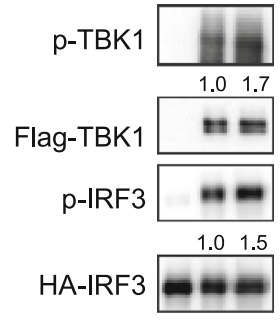
GST-TBK1 - + +
GST-IRF3 + + +
GST-HDAC3
p-TBK1
1.01 .5
TBK1
$\mathbf{u}$
p-IRF3
IRF3 
Figure 4. HDAC3 directly enhances the TBK1 kinase activity via its deacetylase activity. ( $A$ and $B$ ) Luciferase IFN- $\beta$ reporter activity analyzing $(A)$ and Immunoblot analysis of phosphorylated and total TBK1 or IRF3 (B) in HEK293T cells plus RGFP966 in dose $(50,100,200 \mathrm{nmol} / \mathrm{L})$, infected with SeV for 10 h. (C) Immunoblot analysis of HEK293T cells $\left(1.5 \times 10^{6}\right)$ transiently co-transfected for $48 \mathrm{~h}$ with HA-tagged TBK1 together with vector encoding Flag-tagged wild-type HDAC3, HDAC3 (1-316 aa), or HDAC3 (317-428 aa) (above lanes), assessed before (10\% input) or after (IP) immunoprecipitation with anti-Flag antibody. (D) Immunoblot analysis of HEK293T cells $\left(1.5 \times 10^{6}\right)$ transiently co-transfected for $48 \mathrm{~h}$ with HAtagged HDAC3 with vector encoding EGFP-tagged wild-type or mutant TBK1 (above lanes), assessed before (10\% input) or after (Co-IP) Co-immunoprecipitation with the indicated antibodies. (E) TBK1 kinase activity assay combined with purified GST-HDAC3. HEK293T cells $\left(4.0 \times 10^{5}\right)$ was transfected with vector or Flag-tagged TBK1. The expressed proteins immunoprecipitated by Flag tag antibodies and purified GST-HDAC3 or BSA were introduced into a mixture as indicated for TBK1 kinase activity assay. (F) In vitro kinase assay. HEK293T cells $\left(4.0 \times 10^{5}\right)$ were transfected with Flag-tagged TBK1, HA-tagged IRF3 and Flag-tagged HDAC3 separately (left). The expressed proteins were immunoprecipitated by the indicated tag antibodies and incubated together as indicated. The GST-TBK1 purified protein and the peptide of IRF3 full length protein with kinase activity were introduced into a mixture containing recombinant purified HDAC3 (right). Data are representative of three independent experiments. Graphs show mean $\pm \mathrm{SD}$; $n=3$. ${ }^{*} P<0.05$; (Student's $t$-test).

the deacetylation of Lys692 mediated by HDAC3 decided the dimerization of TBK1.

\section{TBK1 phosphorylates HDAC3 to promote its deacetylase activity}

HDAC3 activity is regulated by phosphorylation at Ser424 (Zhang et al., 2005). We suspected that HDAC3 might be phosphorylated as a substrate of TBK1. To test this hypothesis, we used the fusion proteins containing HDAC3 and TBK1 in an in vitro kinase assay. IB analysis of phosphoserine/threonine or the phospho-HDAC3 at Ser424 showed that HDAC3 could be phosphorylated by TBK1 directly (Fig. 8A and 8B). The phosphorylation at Ser424 was enhanced during SeV infection (Fig. 8C). When TBK1 was treated with its inhibitor GSK8612, the phosphorylation of HDAC3 was significantly decreased (Fig. 8D). To determine whether TBK1 influenced the enzymatic activity of HDAC3, we performed an in vitro HDAC3 deacetylation assay using an HDAC activity direct assay kit. As expected, TBK1 markedly increased HDAC3 deacetylation activity (Fig. 8E). Then we analyzed the phosphorylation of HDAC3 in HEK293T cells infected with SeV. The phosphorylation at Ser424 was significantly increased, which was consistent with TBK1 activation. We then analyzed the ratio of HDAC3, TBK1 or IRF3 phosphorylation relative to controls. As shown in Fig. 8F, there was transient increase in HDAC3 phosphorylation at 5-7 $\mathrm{h}$ post $\mathrm{SeV}$ infection, which preceded the phosphorylation of TBK1 or IRF3. These results indicated the TBK1 could phosphorylate HDAC3 to promote its deacetylase activity.

\section{DISCUSSION}

The TBK1-IRF3 axis is critical for the innate immune response. We have shown here that HDAC3 plays an essential role in the innate immune response in vitro and in vivo. HDAC3 deacetylated TBK1 to promote the dimerization and activation of TBK1. However, the acetylation of TBK1 at Lys241 during the early stage of viral infection enhanced the recruitment of IRF3 to TBK1. TBK1 phosphorylated HDAC3 to enhance HDAC3 deacetylation activity and TBK1 activation. Thus, dynamic regulation of TBK1 acetylation and deacetylation plays a key role in the activation of TBK1 and the production of type I IFNs. Our study provides a novel insight into how acetylation tightly regulates TBK1 activation and antiviral responses.

It was reported that HDACs can act as both positive and negative regulators in TLRs- or virus-triggered innate immune responses (Aung et al., 2006; Nusinzon and Horvath, 2006; Halili et al., 2010). HDAC1 and HDAC8 repress the transcription of Ifnb1 mRNA by attenuating the acetylation of histone $\mathrm{H} 3 / \mathrm{H} 4$ in Ifnb1 promoter, while HDAC6 mediates the deacetylation of RIG-I for viral RNA detection and the deacetylation of $\beta$-catenin for IRF3-activated transcription (Liu et al., 2016). Independent of its deacetylase activity, HDAC4 blocks the phosphorylation of IRF3 to repress IRF3-mediated IFN-beta expression (Yang et al., 2018). Here, we provided evidence that HDAC3 positively regulates innate immunity as a deficiency in HDAC3 repressed the production of type I IFNs and antiviral responses both in vitro and in vivo. HDAC3 directly deacetylated TBK1 at Lys241 and Lys692 and activated TBK1 to promote IRF3-activated transcription. HDAC3 is known to shuttle between the nucleus and cytoplasm (Yang et al., 2002; Park et al., 2014). In the nucleus, HDAC3 deacetylates histones and regulates the transcription of different genes (Zhang et al., 2005; Karagianni and Wong, 2009). The HADC3 mutant (HDAC3-NLS), which resides in the nucleus, did not affect the phosphorylation of TBK1 and IRF3, indicating that HDAC3 does not regulate TBK1 activation through the epigenetic modification of chromatin. It has been reported that HDAC9 is involved in the deacetylation of TBK1 ( $\mathrm{Li}$ et al., 2016). HDAC9, a class Ila HDAC, possesses limited deacetylase activity and exhibits activity only when associated with a catalytically active class I HDAC family member, such as HDAC3 (Fischle et al., 2002; Lahm et al., 2007; Chen et al., 2015). Thus, HDAC3 is a key molecule that regulates TBK1 deacetylation to enhance its kinase activity. HDAC3 also positively regulates the IFN-I- 


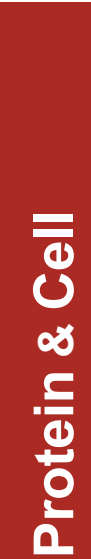

D
A

B IP 导 $\frac{\mathbb{R}}{4}$

Flag-TBK1 +++

Myc-HDAC3 - - +

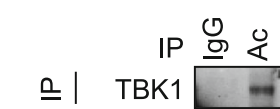

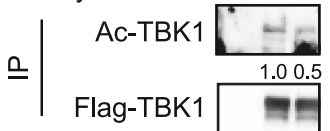

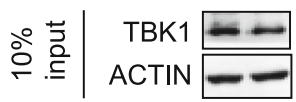

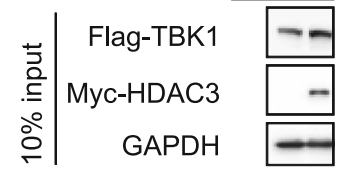

C

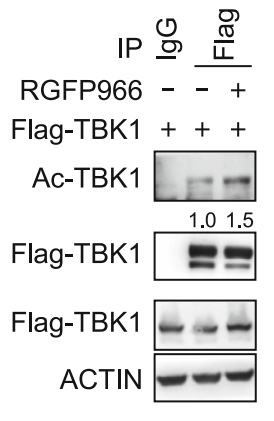

E

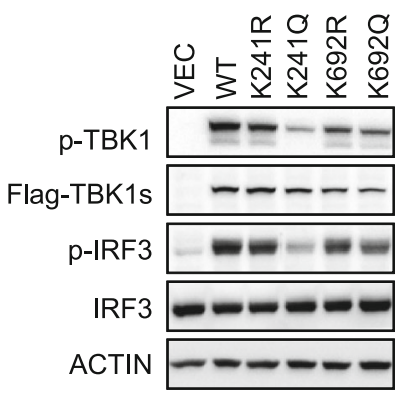

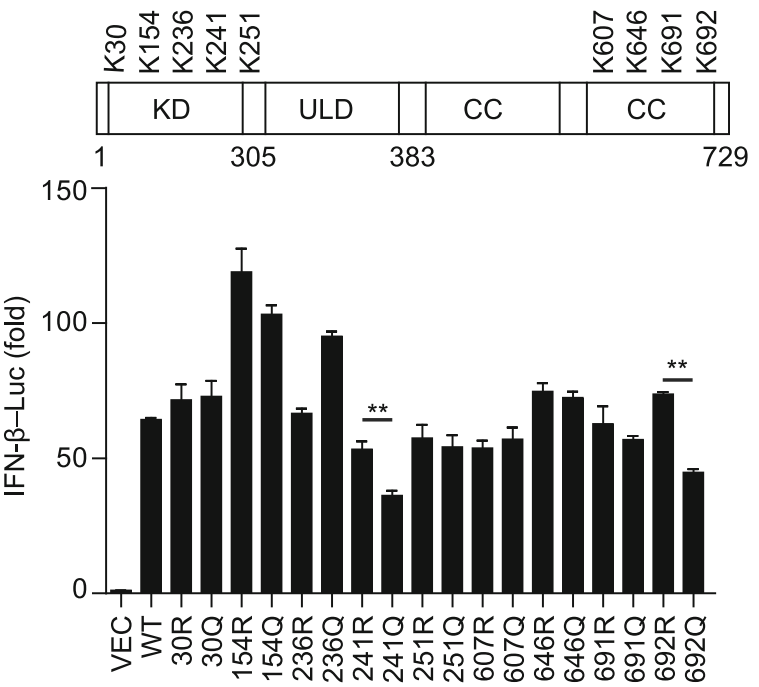

G

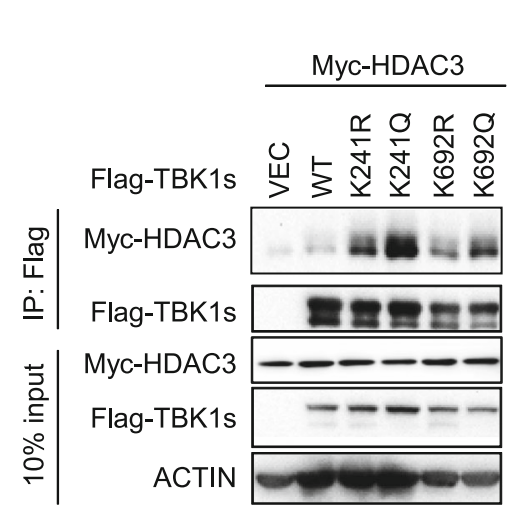

$\mathrm{H}$

IP 可 $\frac{\mathbb{O}}{4}$ 의 $\frac{\mathbb{\pi}}{4}$ shNC shHDAC3

Flag-TBK1

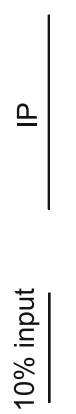

Ac-K241

Ac-K692

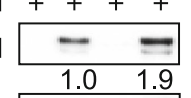

Flag-TBK1
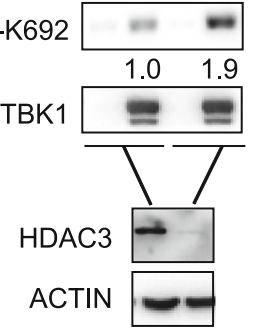

F

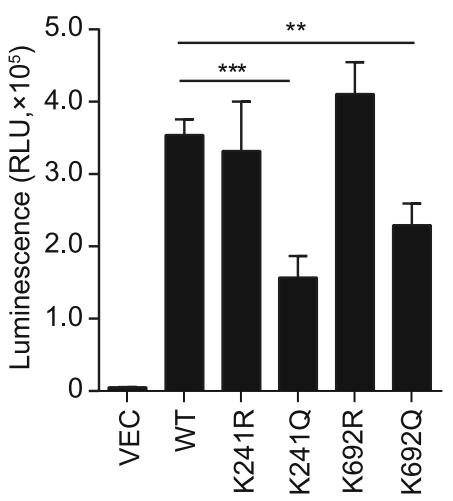

I

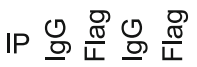

Flag-TBK1 ++++ Myc-HDAC3 $-\quad++$

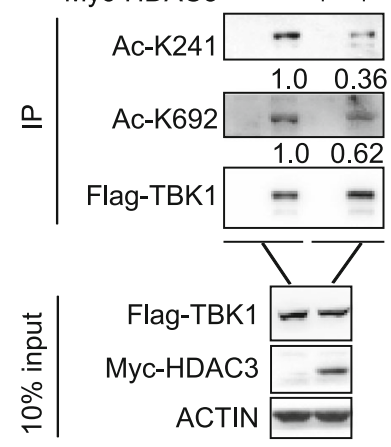


Figure 5. Lys241 and Lys692 deacetylation mediated by HDAC3 are critical for TBK1 kinase activity. (A) Immunoblot analysis of endogenous total TBK1 and acetylated TBK1 (AcTBK1) in HEK293T cells, assessed before (10\% input) or after (IP) immunoprecipitation with IgG (control) or anti-acetyl-lysine. (B) Immunoblot analysis of acetylated TBK1 (Ac-TBK1) in HEK293T cells $\left(1.5 \times 10^{6}\right)$ transiently co-transfected for $48 \mathrm{~h}$ with Flag-tagged TBK1 $(3 \mu \mathrm{g})$ and empty vectors or Myc-tagged HDAC3 $(1 \mu \mathrm{g})$ expression plasmids before co-immunoprecipitation (with anti-Flag or IgG as a control) and immunoblot analysis (with antibody to all acetylated lysine residues, antiFlag, anti-Myc, anti-GAPDH). (C) Effect of HDAC3 specific inhibitor (RGFP966, $100 \mathrm{nmol} / \mathrm{L}$ ) on TBK1 acetylation. Immunoblot analysis of acetylated TBK1 (Ac-TBK1) in HEK293T cells $\left(1.5 \times 10^{6}\right)$, transiently transfected for $48 \mathrm{~h}$ with Flag-tagged TBK1 $(3 \mu \mathrm{g})$ and treated for $24 \mathrm{~h}$ with RGFP966 before immunoprecipitation (with anti-Flag or IgG as a control) and immunoblot analysis (with antibody to all acetylated lysine residues, anti-Flag, anti-ACTIN). (D) Identification of lysine residues in TBK1 in HEK293T HDAC3-deficient cells, by immunoprecipitation combined with mass spectrometry, and Luciferase activity of an IFN- $\beta$ reporter in HEK293T cells transfected with control vector (VEC) or wild-type TBK1 (WT) or mutants TBK1 (30R, 30Q, 154R, 154Q, 236R, 236Q, 241R, 241Q, 251R, 251Q, 607R, 607Q, 646R, 646Q, 691R, 691Q, $692 R, 692 Q$ ). (E) Immunoblot analysis of phosphorylated and total TBK1, IRF3 in HEK293T cells transfected with control vector (VEC) or wild-type TBK1 (WT) or K241R K241Q, K692R, K692Q. (F) TBK1 kinase activity assay. HEK293T TBK1knockout cells $\left(4 \times 10^{5}\right)$ transiently transfected for $48 \mathrm{~h}$ with Flag-tagged empty vector (VEC), wild-type TBK1 (WT) or TBK1 mutants (K241R, K241Q, K692R or K692Q) before immunoprecipitation (with anti-Flag), then according the TBK1 kinase activity kit manufacture. (G) Interaction between HDAC3 and wild type (WT) or TBK1 mutants. Immunoblot analysis of HEK293T cells $\left(1.5 \times 10^{6}\right)$ transiently co-transfected for $48 \mathrm{~h}$ with Myc-tagged HDAC3 and Flag-tagged empty vector, wild type (WT) or TBK1 mutants (K241R, K241Q, K692R, K692Q) (3 $\mu \mathrm{g}$ each) before co-immunoprecipitation (with anti-Flag) and immunoblot analysis were performed with the indicated antibodies. ( $\mathrm{H}$ and $\mathrm{I}$ ) Immunoblot analysis of specific sites of acetylated TBK1 (Ac-K241, Ac-K692) in HDAC3 knockdown HEK293T cells $\left(1.5 \times 10^{6}\right)(\mathrm{H})$ or HDAC3 overexpressed HEK293T cells $\left(1.5 \times 10^{6}\right)(\mathrm{I})$ transiently transfected for $48 \mathrm{~h}$ with Flag-tagged TBK1 ( $3 \mu \mathrm{g}$ each) and empty vectors or Myctagged HDAC3 (1 $\mu \mathrm{g}$ in I) expression plasmids before immunoprecipitation (with anti-Flag or IgG as a control) and immunoblot analysis (with antibody to TBK1 acetylated at Lys241 (K241Ac) or K692Ac, anti-Flag, anti-HDAC3 or anti$\mathrm{Myc}$ ). Data are representative of three independent experiments. Graphs show mean $\pm \mathrm{SD} ; n=3 .{ }^{* \star} P<0.01 ;{ }^{* \star \star} P<0.001$ (Student's $t$-test).

mediated signaling pathway (Feng et al., 2018), which further highlights the functional importance of HDAC3 in the innate immune response and antiviral responses.
As a critical kinase involved in innate immunity, the activity of TBK1 is tightly regulated to maintain immune homeostasis. K63-linked polyubiquitination of TBK1 at Lys30 and Lys401 and autophosphorylation of TBK1 at Ser172 were shown to be critical for its activation (Wang et al., 2009; Li et al., 2011; Ma et al., 2012; Tu et al., 2013; Shevtsova et al., 2016). Modification of TBK1 at Lys694 by the small ubiquitin-like modifier SUMO contributes to the ability of TBK1 to bind to adaptors for the transduction of IFN signaling (Saul et al., 2015). Here, we found that both acetylation and deacetylation were critical for the activation of IRF3 by TBK1, independent of other reported conventional PTMs. During the resting stage, acetylation at Lys241 or Lys692 was able to be detected, but double acetylation of Lys241 and Lys692 was minimal. After induction with stimuli, TBK1 was acetylated at Lys241 and Lys692, and the acetylation level was enhanced at the early stage. We found that the acetylation at Lys241 or Lys692 was independent of one another, as K241R or K692R, mimics of deacetylation, had little effect on the acetylation of the other (Fig. S7A). During induction with virus, the double acetylation of Lys241 and Lys692 was accumulated (Fig. S7B). K241Q, a mimic of acetylation, showed a stronger interaction with IRF3 as compared to the wild-type or K241R, implying that the acetylation of TBK1 at Lys241 enhanced the recruitment of IRF3. However, K241Q had limited kinase activity, which was consistent with the fact that knockdown of HDAC3 repressed IFN production. Thus, the acetylation of TBK1 at Lys241 is an early step before the activation of TBK1. The precise quantitative analysis of TBK1 K241 acetylation is limited by existing technology. It's very difficult to provide the specific data about how much TBK1 K241 was acetylated at the beginning of time course. We only found the acetylation of K241 in uninfected cell was lower compared with Lys241 acetylation at 1-3 $\mathrm{h}$ post $\mathrm{SeV}$ infection (Fig. 6A). Further research is needed to identify which acetylase(s) is involved in the modification and the function of other acetylation sites.

HDAC3-mediated deacetylation of TBK1 at Lys241 and Lys692 occurred during the late stage after SeV challenge (5 hours post-infection). The K241R TBK1 mutant demonstrated a stronger ability to phosphorylate IRF3 in vitro as compared with WT TBK1. Thus, deacetylation of TBK1 at Lys241 enhanced the activation of TBK1. A K692R mutant was able to enhance the dimerization of TBK1 as compared with the K692Q mutant. Thus, HDAC3 mediated the deacetylation of Lys692, which contributed to the dimerization and kinase activity of TBK1. Moreover, the acetylation and deacetylation at Lys241 and Lys692 were coordinated and dynamically regulated, which is essential for the innate immune response.

The phosphorylation of HDAC3 at Ser424 can significantly enhance the deacetylase activity of HDAC3 (Zhang et al., 2005). We found that HDAC3 could be phosphorylated by TBK1. The phosphorylation level of HDAC3 at Ser424 increased at a time point that was consistent with the activation of TBK1. HDAC3 was able to also positively regulate 


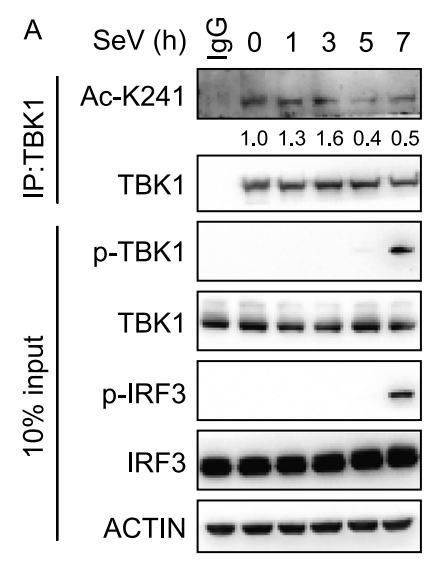

D

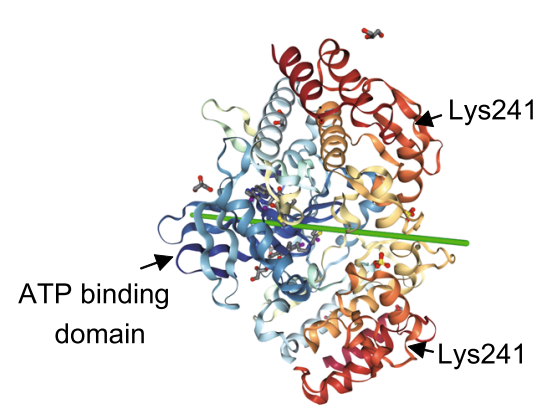

B

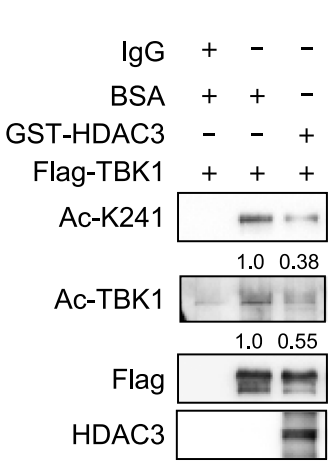

E
C

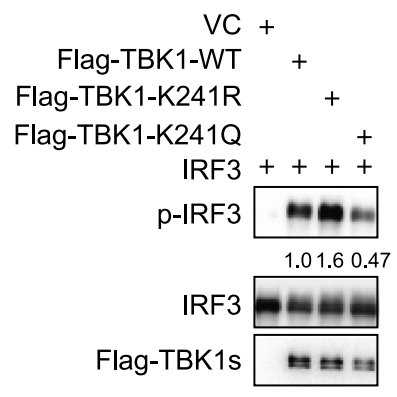

HA-IRF3

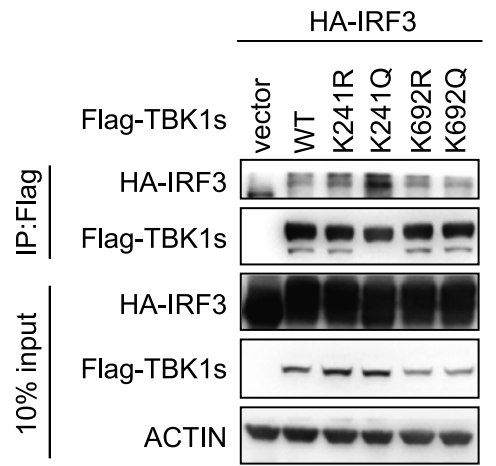

Figure 6. Lys 241 is critical for the interaction between TBK1 and IRF3. (A) Immunoblot analysis of endogenous acetylation of TBK1 at Lys241 (Ac-K241) in HEK293T cells, followed by infection with SeV for 0-7 h, before co-immunoprecipitation (with anti-TBK1 or IgG as a control) and immunoblot analysis with anti-Ac-K241, anti-phosphorylated and total TBK1 or IRF3. (B) In vitro deacetylation assay. The acetyl-modified TBK1 immunoprecipitated and purified recombinant HDAC3 were performed as indicated for deacetylation assay in vitro. Immunoblot analysis with antibody to TBK1 acetylated at Lys241 (Ac-K241), anti-total acetylation, anti-Flag, anti-HDAC3. (C) Effect of TBK1 K241 mutant on kinase assay in vitro. HEK293T cells $\left(4 \times 10^{5}\right)$ were transfected with Flagtagged empty vector (VEC), wild type (WT) or Flag-TBK1-K241R, or Flag-TBK1-K241Q separately. The expressed proteins were immunoprecipitated by the Flag tag antibodies and incubated with recombinant IRF3 protein together as indicated. (D) Structure of TBK1 kinase domain. (E) The interaction between TBK1 K241, K692 mutants and IRF3. Immunoblot analysis of HEK293T cells $\left(1.5 \times 10^{6}\right)$ transiently co-transfected for $48 \mathrm{~h}$ with HA-IRF3 and Flag-tagged empty vector (VEC), wild type (WT) or K241R, K241Q, K692R, K692Q before co-immunoprecipitation (with anti-Flag) and immunoblot analysis were performed with the indicated antibodies. Data are representative of three independent experiments.

the IFN-STAT pathway (Klampfer et al., 2004). The feedback of activation of HDAC3 by TBK1 was able to further enhance IFN production and IFN-STAT activation.

Altogether, we demonstrated not only a new mechanism for the activation of TBK1 and the innate immune response but also that HDAC3 is a precise regulator of the cellular antiviral response.

\section{METHODS}

Reagents and antibodies

Poly I:C (tlrl-pic), 2'3'-cGAMP (tlrl-nacga23-1), CpG ODN (tlrl-20061) were from InvivoGen (France). Lipopolysaccharide (LPS, S1732) was from Beyotime Biotechnology (China). Protein G-agarose (\#16266) used for immunoprecipitation was from Millipore (USA). HDAC3 inhibitor RGFP966 (S7229) was from Selleck (USA). TBK1 inhibitor GSK8612 (\#2361659-62) was from MCE (China). The anti-bodies used in this study are in Table S1. The antibody directed against TBK1 acetylated at Lys241 (TBK1 K241Ac) and Lys692 (TBK1 K692Ac) were customized produced by Abmart (Shanghai, China). Antigenic 11 aa peptide (amino acids 234-244 of TBK1) with acetylated lysine (CYKIIGK(Ac)PSG) and the according control peptide (CYKIIGKPSG) were designed and in vitro synthesized. Antigenic 11 aa peptide (amino acids 685-695 of TBK1) with acetylated lysine (CMTLGMKK(Ac)LKE) and the according control peptide (CMTLGMKKLKE) were designed and in vitro synthesized. The rabbit polyclonal antibodies to the peptides were purified using protein A.

\section{Mice}

All animal procedures in this investigation conform to the Guide for the Care and Use of Laboratory Animals published by the National Institutes of Health (NIH publication 85-23, revised in 1996) and the approved regulations were set by the Laboratory Animal Care 


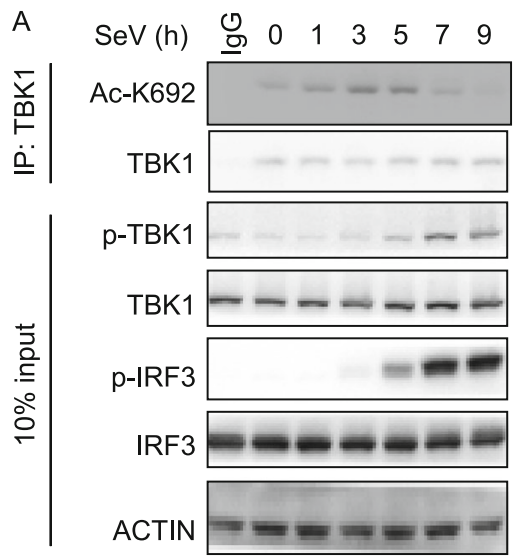

D

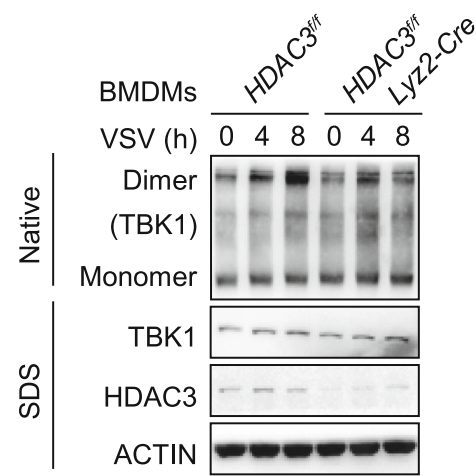

C

$\begin{array}{rcccc}\text { IP } & \text { 음 } & \frac{\pi}{4} & \text { 은 } & \frac{\pi}{4} \\ \text { HA-TBK1 } & + & + & + & + \\ \text { Flag-TRAF3 } & + & + & + & + \\ \text { Myc-HDAC3 } & - & - & + & +\end{array}$

ㅇ

HA-TBK1

Flag-TRAF3

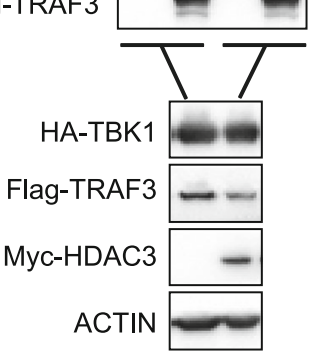

B

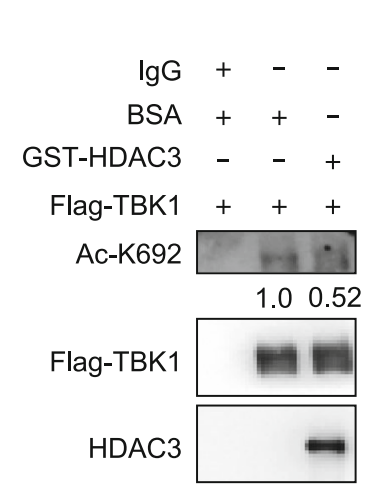

IP 음 $\frac{\mathbb{D}}{4}$ 음 $\frac{\text { O }}{4}$

$\mathrm{HA}-\mathrm{AZI} 2++++$

Flag-TBK1 ++++

Myc-HDAC3 $-\quad++$

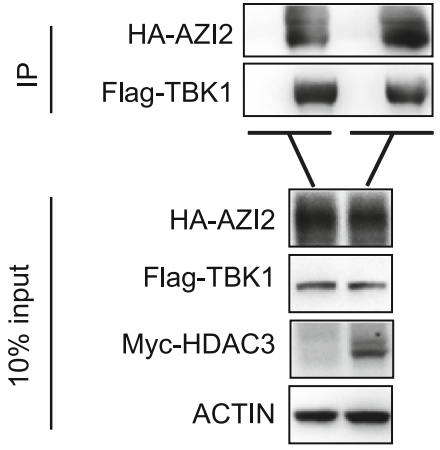

E

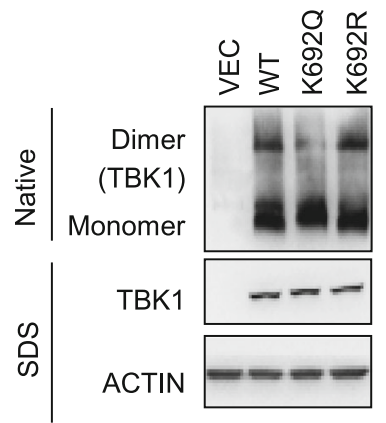

Figure 7. Lys692 deacetylation controls the dimerization of TBK1. (A) Immunoblot analysis of endogenous acetylation of TBK1 at Lys692 (Ac-K692) in HEK293T cells $\left(1.5 \times 10^{7}\right.$ ), followed by infection with SeV for 0-9 h, before co-immunoprecipitation (with antiTBK1 or IgG as a control) and immunoblot analysis with anti-Ac-K692, anti-phosphorylated and total TBK1or IRF3. (B) In vitro deacetylation assay. Assays were performed as in Fig. 6B. Immunoblot analysis with antibody to TBK1 acetylated at Lys692 (AcK692), anti-Flag, anti-HDAC3. (C) Effect of HDAC3 overexpression on TRAF3-TBK1, AZI2-TBK1 association. Immunoblot analysis of HEK293T cells $\left(1.5 \times 10^{6}\right)$ transiently co-transfected for $48 \mathrm{~h}$ with the indicated plasmids before co-immunoprecipitation (with antiFlag or IgG as a control) and immunoblot analysis were performed with the indicated antibodies. (D) Immunoblot analysis of TBK1 in dimerization in $H D A C 3^{\text {fl/fl }}$ or HDAC3 $3^{\text {fl/fl}}$ Lyz2-Cre BMDMs and left infected with VSV for the indicated times, followed by native PAGE. (E) Immunoblot analysis of TBK1 in dimer or monomer form in HEK293T TBK1-knockout cells $\left(4 \times 10^{5}\right)$, transiently transfected for 48 h with Flag-tagged empty vector (VEC), wild type (WT) or TBK1 mutants (K692Q or K692R), followed by native PAGE. Data are representative of three independent experiments.

Committee at Wuhan Institute of Virology, Chinese Academy of Sciences (CAS) (permit WIVA02201703). Lyz2-Cre mice on the C57BL/6 background were from Model Animal Research Center of Najing University. $H D A C 3^{f l f f}$ mice on the C57BL/6 background were provided by Prof. Yujun Shi (Sichuan University). All of the mice were bred and maintained under specific-pathogen free animal facility in Wuhan Institute of Virology, CAS.
Cells and virus

All cell cultures were maintained in a humidified atmosphere at $37^{\circ} \mathrm{C}$ with $5 \% \mathrm{CO}_{2}$. The HEK293T, Raw264.7, THP-1 and Vero cell were from American Type Culture Collection (ATCC), and cultured in Dulbecco's modified Eagle medium (DMEM) (Invitrogen, USA) supplemented with $2 \mathrm{mmol} / \mathrm{L}$ L-glutamine, nonessential amino acids, $10 \%$ fetal bovine serum (FBS) (Invitrogen), and 1\% penicillin-streptomycin (Life Technologies, USA). THP-1 cells were cultured in RPMI 1640 
A

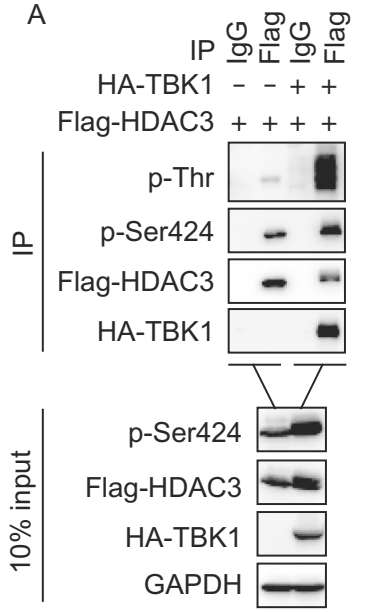

E

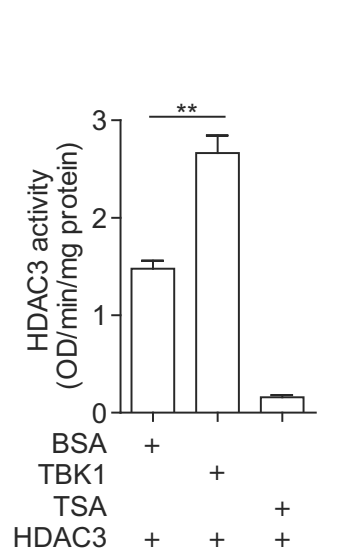

F

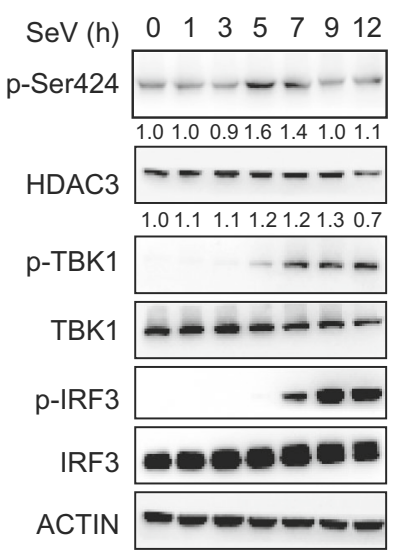

B

$B$

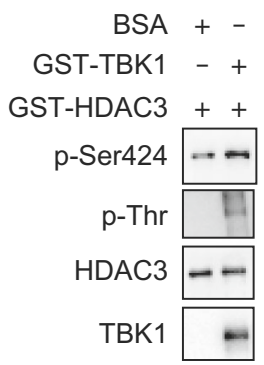

C

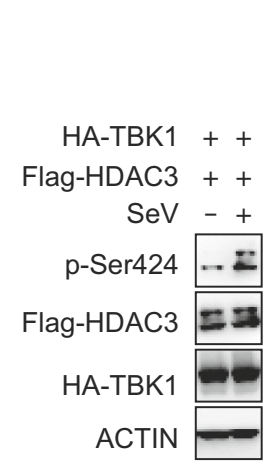

D

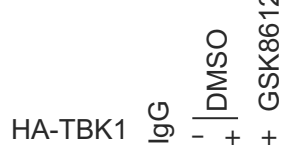
Flag-HDAC3 ++++

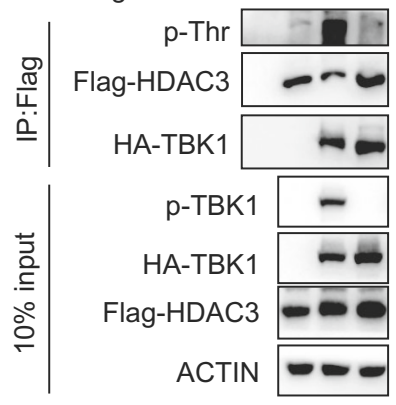

Figure 8. TBK1 phosphorylates HDAC3 to promote its eacetylase activity. (A) Immunoblot analysis of phosphorylated HDAC3 by TBK1 in HEK293T cells $\left(1.5 \times 10^{6}\right)$ transiently co-transfected for $48 \mathrm{~h}$ with Flag-tagged HDAC3 and empty vectors or HAtagged TBK1 expression plasmids before co-immunoprecipitation (with anti-Flag or lgG as a control) and immunoblot analysis (with antibody to anti-Phospho-HDAC3 (Ser424), anti-Phospho-Threonine, anti-HA or anti-Flag). (B) In vitro kinase assay of phosphorylated HDAC3. The peptide of HDAC3 full length protein as the substrate and combinations of BSA or recombinant protein TBK1 were introduced as indicated and the immunoblot analysis with antibody to anti-Phospho-HDAC3 (Ser424), antiPhospho-Threonine, anti-HDAC3 or anti-TBK1. (C) Immunoblot analysis of HEK293T cells $\left(2 \times 10^{5}\right)$ transiently co-transfected for $48 \mathrm{~h}$ with HA-tagged TBK1 $(1 \mu \mathrm{g})$ and Flag-tagged HDAC3 $(300 \mathrm{ng})$, followed by infection with or without SeV for $6 \mathrm{~h}$. (D) HDAC3 was phosphorylated by TBK1. HEK293T cells $\left(1.5 \times 10^{6}\right)$ were transiently co-transfected for $48 \mathrm{~h}$ with Flag-tagged HDAC3 and empty vectors or HA-tagged TBK1 expression plasmids, and treated with GSK8612 for $1 \mathrm{~h}$ before co-immunoprecipitation (with anti-Flag or IgG as a control) and the immunoblot analysis with antibody to anti-Phospho-Threonine, anti-HA, anti-Flag or anti-phospho-TBK1. (E) Analysis of HDAC3 enzymatic activity in the presence of recombinant TBK1 protein or TSA (negative control). (F) Immunoblot analysis of phosphorylated and total HDAC3, TBK1 or IRF3 and $\beta$-actin of HEK293T cells infected with SeV for 0-12 h (left) and densitometric analysis (right). Data are representative of three independent experiments. Graphs show mean $\pm \mathrm{SD} ; n=3$. ${ }^{* \star} P<0.01$ (Student's $t$-test).

(Hyclone, USA) supplemented with 10\% fetal bovine serum (FBS) (Invitrogen), and 1\% penicillin-streptomycin (Life Technologies, USA).

Sendai virus (SeV) and vesicular stomatitis virus VSV-GFP were provided by Prof. Hanzhong Wang, HSV-1 with GFP was provided by Prof. Chunfu Zheng. All virus were amplified and titrated were as previously described. Cells were infected with SeV (1 M.O.I.), VSV (1 M.O.I) or HSV-1 (10 M.O.I.) for the indicated time. Cells were stimulated with poly(l:C) $(50 \mu \mathrm{g} / \mathrm{mL})$, LPS (100 $\mathrm{ng} / \mathrm{mL})$, cGAMP $(1 \mu \mathrm{g} / \mathrm{mL})$, CPG ODN $(1 \mu \mathrm{g} / \mathrm{mL})$ for the indicated time.

\section{CRISPR-Cas9 knockout}

Double-stranded oligonucleotides corresponding to the target sequences were cloned into the lenti-CRISPR-V2 vector and cotransfected packaging plasmids into HEK293 cells. $48 \mathrm{~h}$ after 
transfection, the viruses were harvested, ultra-filtrated $(0.22 \mathrm{~mm}$ filter, Millipore) and used to infect HEK293T or THP1 cells in the presence of polybrene $(8 \mu \mathrm{g} / \mathrm{mL})$. The infected cells were selected with puromycin $(2 \mu \mathrm{g} / \mathrm{mL})$ for at least 5 days. Human HDAC3 and TBK1 sgRNA targeting sequences were in Tables S4.

\section{Isolation of macrophages}

BMDMs were isolated from the mouse tibia and femur of wild-type and HDAC3 deficient mice and cultured in $10-\mathrm{cm}$ Petri dish at $37^{\circ} \mathrm{C}$ for 6 days. $10 \mathrm{~mL}$ RPMI medium 1640 (supplemented with $10 \%$ FBS, L-glutamine, and 30\% L929 supernatant) was added at day 4 .

\section{Plasmid constructs and transfection}

Mammalian expression plasmids for HA-, Flag-, Myc-tagged HDAC3 and its Flag-tagged deletion mutants were constructed by standard molecular biology techniques. Mammalian expression plasmids for HA- or Flag-tagged TBK1 and all Flag-tagged site-directed mutagenesis of TBK1 were amplified and inserted into the $\mathrm{pXJ40}$ vectors. All constructs were confirmed by sequencing and a complete list of primers was provided in the supplementary materials (Table S2). Mammalian expression plasmids for RIG-I, VISA, IRF3-5D were provided by Prof. Hongbing Shu. IFN- $\beta$, Interferon-stimulated response element (ISRE) promoter luciferase reporter plasmids were purchased from Clontech (USA). pRL-TK was purchased from Promega (USA). The plasmids for EGFP-C1-TBK1 deletion mutants, HA-tagged TANK, HA-tagged AZI2 were constructed by our lab. Plasmids were transiently transfected into HEK293T cells with using Lipofectamine 2000 reagents (Invitrogen, USA) following the manufacturer instructions.

\section{Luciferase assays}

HEK293T $\left(1 \times 10^{5}\right)$ in 24-well plates were transfected with IFN- $\beta$ or ISRE-firefly luciferase reporter (100 ng) and Renilla luciferase reporter $(10 \mathrm{ng})$ together with HDAC3 or empty control plasmid in dose. $24 \mathrm{~h}$ later, the cells were stimulated with virus for $10 \mathrm{~h}$, and luciferase activities were measured with Dual-Glo® Luciferase Assay System (E1960, Promega, USA). Data were normalized by calculating the ratio between firefly luciferase activity and Renilla luciferase activity.

\section{ELISA}

Secreted cytokines in cell culture supernatants or sera from virus infected-mice were analyzed using human IFN- $\beta$ (CUSABIO, China), mouse IFN- $\beta$ (Biolegend, China) ELISA kits according to the manufacturer's instructions.

\section{RNA-mediated interference and lentiviral transduction}

HEK293T $\left(4 \times 10^{5}\right)$ in 6-well plates were transfected with siRNA $(20 \mathrm{nmol} / \mathrm{L})$ by using Lipofectamine RNAiMAX reagent (Invitrogen, USA). The following sequences targeting for HDAC3 were designed and synthesized by Qiagen (Germany). The lentiviruses were produced in 293T cells by co-transfection of the shNC or shHDAC3 and packaging vectors psPAX2 and pMD2.G (Bought from addgene), (shRNAs were cloned into the lentivirus vector pLKO.1 plasmid).
$48 \mathrm{~h}$ or $72 \mathrm{~h}$ post-transfection, the supernatant was collected and applied to infect target cells, followed by cell selection through puromycin $(1 \mu \mathrm{g} / \mathrm{mL})$. The information of the sequences was in Table S4.

\section{RNA quantification}

Total RNA was extracted with Trizol reagent following the manufacturer's protocols (Invitrogen, USA). Specific mRNAs were quantified by one-step real-time RT-PCR using the QuantiFast SYBR Green RT-PCR kit (Qiagen, Germany). The data were normalized to the expression of the $\beta$-actin for each individual sample. The $2^{-\Delta \Delta C t}$ method was used to calculate relative expression changes. The primer sequences for quantitative RT-PCR are provided in Table S3.

Co-immunoprecipitation and immunoblot analysis

For co-immunoprecipitation and immunoblot analysis, cells were lysed with lysis buffer $(50 \mathrm{mmol} / \mathrm{L}$ Tris, $\mathrm{pH} 7.5,1 \mathrm{mmol} / \mathrm{L}$ EGTA, $1 \mathrm{mmol} / \mathrm{L}$ EDTA, $1 \%$ Triton X-100, $150 \mathrm{mmol} / \mathrm{L} \mathrm{NaCl}, 100 \mu \mathrm{mol} / \mathrm{L}$ phenylmethylsulfonyl fluoride (PMSF), and a protease inhibitor cocktail (Complete Mini, Roche, Swiss) for $30 \mathrm{~min}$ in $4{ }^{\circ} \mathrm{C}$. Cell lysates were centrifuged at $13,000 \times g$ for $10 \mathrm{~min}$ at $4{ }^{\circ} \mathrm{C}$ and quantified using the Bradford method (\#500-0006, Bio-Rad, USA). For Western blot, the supernatants were recovered and boiled in $5 \times$ loading buffer. For immunoprecipitation, the supernatants were collected and incubated with protein $\mathrm{G}$ agarose beads recovered and mixed with specific antibodies for overnight with rotation at $4{ }^{\circ} \mathrm{C}$. Protein $\mathrm{G}$ agarose-bound immune complexes were collected by centrifugation at 5,000 $\times g$ for $1 \mathrm{~min}$, washed at least five times with IP buffer, and boiled in $2 \times$ loading buffer. Samples were separated by SDS-PAGE, electro-transferred to nitrocellulose filter membranes (Millipore, USA) and then blocked for $1 \mathrm{~h}$ with $5 \%$ nonfat milk solution, followed by blotting with the primary antibodies. The proteins were visualized using suitable HRP-conjugated secondary antibodies (Jackson Immuno Research, USA) and SuperSignal-Femto chemiluminescent substrate (Pierce, USA).

\section{In vitro kinase assay}

HEK293T $\left(1.5 \times 10^{6}\right)$ cells were transfected with indicated plasmid separately. The expressed proteins were immunoprecipitated by the indicated tag antibodies. The immune-complexes were washed twice with $1 \times$ cell lysis buffer (\#9803s, CST, USA) and then twice with $1 \times$ kinase buffer (\#9802, CST, USA). Kinase reactions were performed by incubation of immune-complexes or purified proteins as indicated with $1 \times$ kinase buffer, $10 \mathrm{mmol} / \mathrm{L}$ ATP (\#9804, CST, USA) and at $30{ }^{\circ} \mathrm{C}$ for $90 \mathrm{~min}$ in $50 \mu \mathrm{L}$ reaction mixture. Samples were separated by SDS-PAGE, and analyzed by immunoblotting with anti-phospho-IRF3 and anti-phospho-TBK1.

\section{In vitro deacetylation assay}

HEK293T $\left(4 \times 10^{5}\right)$ cells in 6-well plates were transfected with Flagtagged vector, or WT, or TBK1 mutants (241R, 241Q, 692R, 692Q). The expressed proteins were immunoprecipitated by Flag antibody. The immunoprecipitate were washed with deacetylation buffer (50 mmol/L Tris- $\mathrm{HCl}(\mathrm{pH} 8.0), 50 \mathrm{mmol} / \mathrm{L} \mathrm{NaCl}, 10 \%$ glycerol, 0.5 $\mathrm{mmol} / \mathrm{L}$ EDTA, $1 \mathrm{mmol} / \mathrm{L}$ dithiothreitol, $10 \mathrm{mmol} / \mathrm{L}$ sodium buytrate 
and $1 \mathrm{mmol} / \mathrm{L}$ phenylmethylsulfonyl fluoride). Deacetylation reactions were performed by incubation of immunoprecipitate and purified GST-tagged HDAC3 protein as indicated with $1 \times$ deacetylation buffer, at $37^{\circ} \mathrm{C}$ for $90 \mathrm{~min}$ in $50 \mu \mathrm{L}$ reaction mixture. Samples were separated by SDS-PAGE, and analyzed by immunoblotting with K241Ac, K692Ac antibodies.

\section{Mass spectrometry}

HEK293T $\left(1 \times 10^{7}\right)$ cells were infected with $\mathrm{SeV}$ and then lysed for immunoprecipitation with antibody to acetyl (or with IgG as a control). After Commassie Blue staining, the TBK1 specific band with intensive signal compared with lgG control were cut and then used as the sample for mass spectrometry.

\section{TBK1 kinase activity assay}

HEK293T TBK1 KO $\left(4 \times 10^{5}\right)$ cells in 6-well plates were transfected with Flag-tagged vector, or WT, or TBK1 mutants $(241 \mathrm{R}, 241 \mathrm{Q}$, 692R, 692Q). $48 \mathrm{~h}$ after transfection, the cells were lysed for immunoprecipitation with antibody to Flag. The TBK1 kinase activity was analyzed using TBK1 Kinase Enzyme System (V8291, Promega, USA). TBK1 kinase activity assay according to the manufacturer's instructions.

\section{IRF3 and TBK1 dimer assay}

Native PAGE was performed with an $8 \%$ acrylamide gel without SDS. The gel was pre-run for $60 \mathrm{~min}$ at $200 \mathrm{~V}$ on ice with $25 \mathrm{mmol} / \mathrm{L}$ Tris- $\mathrm{HCl}(\mathrm{pH} 8.4)$ and $192 \mathrm{mmol} / \mathrm{L}$ glycine with or without $0.5 \%$ deoxycholate in the cathode buffer and anode buffer, respectively. Samples in the $5 \times$ loading buffer $(50 \mathrm{mmol} / \mathrm{L}$ Tris- $\mathrm{HCl}, \mathrm{pH} 6.8$, $0.002 \%$ Bromphenol Blue, and $15 \%$ glycerol) were applied on the gel and underwent electrophoresis for $60 \mathrm{~min}$ at $200 \mathrm{~V}$ on ice, followed by immunoblot analysis.

\section{Statistical analysis}

Differences between groups were evaluated using the two-tailed, unpaired Student's $t$-test available in the GraphPad Prism 5 software package (GraphPad Software, Inc.). Coprecipitation efficiency and fluorescence images were analyzed using ImageJ $(\mathrm{NIH})$. $P$-values were calculated, and statistical significance was reported as highly significant with ${ }^{*} P<0.05$ ). Analytic results are presented as mean \pm SD.

\section{ACKNOWLEDGEMENTS}

We thank LetPub (www.letpub.com) for its linguistic assistance during the preparation of this manuscript. Thanks for the core facilities center of Wuhan Institute of Virology for technical help. We thank Mr. He Zhao in animal experiment center for animal experiment help. We are grateful to Prof. Yujun Shi (Sichuan University) for $H D A C 3^{\text {fllfl }}$ mice.

This study was supported by the National Key Research and Development Program of China (2018YFA0507201 to X.C. and 2018YFA0507202 to Y.Z.). This study was supported by the Youth Innovation Promotion Association.

\section{AUTHOR CONTRIBUTIONS}

The study was designed by J.Z.C. and X.W.C.; J.L.T. performed the experiments; J.Z.C. J.L.T. and X.W.C. wrote the paper; B.Z. and Y.Y.W. offered experimental advice; all authors analyzed data.

\section{ABBREVIATIONS}

AZI2, 5-azacytidine-induced protein 2; BMDMs, bone marrow derived macrophages; cGAMP, cyclic GMP-AMP; CpG ODN, CpG oligodeoxynucleotide; HDAC3, histone deacetylase 3; HDACs, histone deacetylases; HSV-1, herpes simplex virus-1; IFNs, interferons; IRF3, interferon-regulatory factors 3; ISRE, IFN-stimulated regulatory element; LPS, lipopolysaccharide; NLS, nuclear localization signal; PAMPs, pathogen-associated molecular patterns; poly(I:C), polyinosinic-polycytidylic acid; PRRs, pattern recognition receptors; PTMs, post-translational modifications; RIG-I, retinoic acid-inducible gene I; SeV, sendai virus; STAT, signal transducing activator of transcription; TANK, TRAF family member-associated NF-kappa-B activator; TBK1, TANK-binding kinase 1; TLRs, Toll-like receptors; TRAF3, TNF receptor associated factor 3; TRAF6, TNF receptor associated factor 6 ; VISA, virus-induced-signaling adapter; VSV, vesicular stomatitis virus

\section{COMPLIANCE WITH ETHICS GUIDELINES}

Jie-lin Tang, Qi Yang, Chong-hui Xu, He Zhao, Ya-ling Liu, Can-yu Liu, Yuan Zhou, Dong-wei Gai, Rong-juan Pei, Yun Wang, Xue Hu, Bo Zhong, Yan-yi Wang, Xin-wen Chen, Ji-zheng Chen declare that they have no conflict of interest.

All institutional and national guidelines for the care and use of laboratory animals were followed.

\section{OPEN ACCESS}

This article is licensed under a Creative Commons Attribution 4.0 International License, which permits use, sharing, adaptation, distribution and reproduction in any medium or format, as long as you give appropriate credit to the original author(s) and the source, provide a link to the Creative Commons licence, and indicate if changes were made. The images or other third party material in this article are included in the article's Creative Commons licence, unless indicated otherwise in a credit line to the material. If material is not included in the article's Creative Commons licence and your intended use is not permitted by statutory regulation or exceeds the permitted use, you will need to obtain permission directly from the copyright holder. To view a copy of this licence, visit http:// creativecommons.org/licenses/by/4.0/.

\section{REFERENCES}

Akira S, Uematsu S, Takeuchi O (2006) Pathogen recognition and innate immunity. Cell 124:783-801

An T, Li S, Pan W, Tien P, Zhong B, Shu HB, Wu S (2015) DYRK2 negatively regulates type I interferon induction by promoting 
TBK1 degradation via Ser527 phosphorylation. PLoS Pathog 11: e1005179

Aung HT, Schroder K, Himes SR, Brion K, van Zuylen W, Trieu A, Suzuki H, Hayashizaki Y, Hume DA, Sweet MJ et al (2006) LPS regulates proinflammatory gene expression in macrophages by altering histone deacetylase expression. FASEB J 20:1315-1327

Cao W, Bao C, Padalko E, Lowenstein CJ (2008) Acetylation of mitogen-activated protein kinase phosphatase-1 inhibits Toll-like receptor signaling. J Exp Med 205:1491-1503

Chen J, Wang N, Dong M, Guo M, Zhao Y, Zhuo Z, Zhang C, Chi X, Pan $Y$, Jiang $J$ et al (2015) The metabolic regulator histone deacetylase 9 contributes to glucose homeostasis abnormality induced by hepatitis $\mathrm{C}$ virus infection. Am Diabetes Assoc 64:4088

Chini CC, Escande C, Nin V, Chini EN (2010) HDAC3 is negatively regulated by the nuclear protein DBC1. J Biol Chem 285:4083040837

Dai J, Huang YJ, He X, Zhao M, Wang X, Liu ZS, Xue W, Cai H, Zhan XY, Huang SY et al (2019) Acetylation blocks cGAS activity and inhibits Self-DNA-induced autoimmunity. Cell 176(14471460):e1414

Feng Q, Miao Y, Ge J, Yuan Y, Zuo Y, Qian L, Liu J, Cheng Q, Guo T, Zhang $L$ et al (2018) ATXN3 positively regulates type I IFN antiviral response by deubiquitinating and stabilizing HDAC3. J Immunol 201:675-687

Fischle W, Dequiedt F, Hendzel MJ, Guenther MG, Lazar MA, Voelter W, Verdin E (2002) Enzymatic activity associated with class II HDACs is dependent on a multiprotein complex containing HDAC3 and SMRT/N-CoR. Mol Cell 9:45

Fitzgerald KA, McWhirter SM, Faia KL, Rowe DC, Latz E, Golenbock DT, Coyle AJ, Liao SM, Maniatis T (2003) IKKepsilon and TBK1 are essential components of the IRF3 signaling pathway. Nat Immunol 4:491-496

Halili MA, Andrews MR, Labzin LI, Schroder K, Matthias G, Cao C, Lovelace E, Reid RC, Le GT, Hume DA et al (2010) Differential effects of selective HDAC inhibitors on macrophage inflammatory responses to the Toll-like receptor 4 agonist LPS. J Leukoc Biol 87:1103-1114

Honda K, Taniguchi T (2006) IRFs: master regulators of signalling by toll-like receptors and cytosolic pattern-recognition receptors. Nat Rev Immunol 6:644-658

Karagianni P, Wong J (2009) HDAC3: taking the SMRT-N-CoRrect road to repression. Oncogene 26:5439

Klampfer L, Huang J, Swaby LA, Augenlicht L (2004) Requirement of histone deacetylase activity for signaling by STAT1. J Biol Chem 279:30358-30368

Lahm A, Paolini C, Pallaoro M, Nardi MC, Jones P, Neddermann P, Sambucini S, Bottomley MJ, Lo Surdo P, Carfi A et al (2007) Unraveling the hidden catalytic activity of vertebrate class Ila histone deacetylases. Proc Natl Acad Sci USA 104:17335-17340

Larabi A, Devos JM, Ng SL, Nanao MH, Round A, Maniatis T, Panne D (2013) Crystal structure and mechanism of activation of TANKbinding kinase 1. Cell Rep 3:734-746
Li S, Wang L, Berman M, Kong YY, Dorf ME (2011) Mapping a dynamic innate immunity protein interaction network regulating type I interferon production. Immunity 35:426-440

Li X, Zhang Q, Ding Y, Liu Y, Zhao D, Zhao K, Shen Q, Liu X, Zhu X, $\mathrm{Li} N$ et al (2016) Methyltransferase Dnmt3a upregulates HDAC9 to deacetylate the kinase TBK1 for activation of antiviral innate immunity. Nat Immunol 17:806-815

Liu HM, Jiang F, Loo YM, Hsu S, Hsiang TY, Marcotrigiano J, Gale M Jr (2016) Regulation of retinoic acid inducible gene-I (RIG-I) activation by the histone deacetylase 6. EBioMedicine 9:195-206

Liu S, Chen S, Li X, Wu S, Zhang Q, Jin Q, Hu L, Zhou R, Yu Z, Meng $F$ et al (2017) Lck/Hck/Fgr-mediated tyrosine phosphorylation negatively regulates TBK1 to restrain innate antiviral responses. Cell Host Microbe 21(754-768):e755

Ma X, Helgason E, Phung QT, Quan CL, lyer RS, Lee MW, Bowman KK, Starovasnik MA, Dueber EC (2012) Molecular basis of tankbinding kinase 1 activation by transautophosphorylation. Proc Natl Acad Sci USA 109:9378-9383

Medzhitov R (2007) Recognition of microorganisms and activation of the immune response. Nature 449:819-826

Meng J, Liu X, Zhang P, Li D, Xu S, Zhou Q, Guo M, Huai W, Chen $X$, Wang $Q$ et al (2016) Rb selectively inhibits innate IFN-beta production by enhancing deacetylation of IFN-beta promoter through HDAC1 and HDAC8. J Autoimmun 73:42-53

Mihaylova MM, Vasquez DS, Ravnskjaer K, Denechaud PD, Yu RT, Alvarez JG, Downes M, Evans RM, Montminy M, Shaw RJ (2011) Class Ila histone deacetylases are hormone-activated regulators of FOXO and mammalian glucose homeostasis. Cell 145:607-621

Mowen KA, David M (2014) Unconventional post-translational modifications in immunological signaling. Nat Immunol 15:512520

Nusinzon I, Horvath CM (2006) Positive and negative regulation of the innate antiviral response and beta interferon gene expression by deacetylation. Mol Cell Biol 26:3106-3113

Park H, Kim Y, Park D, Jeoung D (2014) Nuclear localization signal domain of HDAC3 is necessary and sufficient for the expression regulation of MDR1. BMB Rep 47:342-347

Saul VV, Niedenthal R, Pich A, Weber F, Schmitz ML (2015) SUMO modification of TBK1 at the adaptor-binding C-terminal coiled-coil domain contributes to its antiviral activity. Biochim Biophys Acta 1853:136-143

Shevtsova AS, Motuzova OV, Kuragina VM, Akhmatova NK, Gmyl LV, Kondrat'eva YI, Kozlovskaya LI, Rogova YV, Litov AG, Romanova LI et al (2016) Lethal experimental tick-borne encephalitis infection: influence of two strains with similar virulence on the immune response. Front Microbiol 7:2172

Smale ST, Tarakhovsky A, Natoli G (2014) Chromatin contributions to the regulation of innate immunity. Annu Rev Immunol 32:489511

Song G, Liu B, Li Z, Wu H, Wang P, Zhao K, Jiang G, Zhang L, Gao C (2016) E3 ubiquitin ligase RNF128 promotes innate antiviral immunity through K63-linked ubiquitination of TBK1. Nat Immunol $17: 1342-1351$ 
Suhara W, Yoneyama M, Iwamura T, Yoshimura S, Tamura K, Namiki H, Aimoto S, Fujita T (2000) Analyses of virus-induced homomeric and heteromeric protein associations between IRF-3 and coactivator $\mathrm{CBP} / \mathrm{p} 300$. J Biochem 128:301-307

Takeuchi O, Akira S (2010) Pattern recognition receptors and inflammation. Cell 140:805-820

Tu D, Zhu Z, Zhou AY, Yun CH, Lee KE, Toms AV, Li Y, Dunn GP, Chan $E$, Thai $T$ et al (2013) Structure and ubiquitination-dependent activation of TANK-binding kinase 1. Cell Rep 3:747-758

Wang C, Chen T, Zhang J, Yang M, Li N, Xu X, Cao X (2009) The E3 ubiquitin ligase Nrdp1 'preferentially' promotes TLR-mediated production of type I interferon. Nat Immunol 10:744-752
Yang Q, Tang J, Pei R, Gao X, Guo J, Xu C, Wang Y, Wang Q, Wu C, Zhou $Y$ et al (2018) Host HDAC4 regulates the antiviral response by inhibiting the phosphorylation of IRF3. J Mol Cell Biol 11:158

Yang WM, Tsai SC, Wen YD, Fejer G, Seto E (2002) Functional domains of histone deacetylase-3. J Biol Chem 277:9447-9454

Zhang X, Ozawa Y, Lee H, Wen YD, Tan TH, Wadzinski BE, Seto E (2005) Histone deacetylase 3 (HDAC3) activity is regulated by interaction with protein serine/threonine phosphatase 4 . Genes Dev 19:827-839 